\title{
On smooth time functions
}

\author{
Albert Fathi And Antonio Siconolfi \\ Version after Referee's Corrections August 12, 2011
}

\begin{abstract}
We are concerned with the existence of smooth time functions on connected time-oriented Lorentzian manifolds. The problem is tackled in a more general abstract setting, namely in a manifold $M$ where is just defined a field of tangent convex cones $\left(\mathcal{C}_{x}\right)_{x \in M}$ enjoying mild continuity properties. Under some conditions on its integral curves, we will construct a time function.

Our approach is based on the definition of an intrinsic length for curves indicating how a curve is far from being an integral trajectory of $\mathcal{C}_{x}$. We find connections with topics pertaining to Hamilton-Jacobi equations, and make use of tools and results issued from weak KAM theory.
\end{abstract}

\section{Introduction}

In this paper we are concerned with the existence of smooth time functions on connected time-oriented Lorentzian manifolds, space-times for short, under appropriate assumptions relative to the causal structure.

As a matter of fact our analysis takes place, and so our results are valid, in a more general environment. What we really need as underlying space is a (connected, paracompact, smooth) manifold $M$ on which is defined a multivalued vector field $x \mapsto \mathcal{C}_{x} \subset T_{x} M$, with convex cones as values, a cone structure for short. Here and throughout the paper cone means a closed convex cone, with vertex at 0 , not containing any complete affine line and whose interior is not empty.

We will require quite mild continuity properties to hold for $\mathcal{C}$, as well as some specific conditions on its integral curves, namely the (Lipschitz) curves $\xi$ with $\dot{\xi}(t) \in \mathcal{C}_{\xi(t)}$. Framing the problem in this broader setting allows us to better understand the proof of existence of smooth time functions in Lorentzian geometry.

For a space-time with Lorentzian metric $h$ of signature $(+, \cdots,+,-)$, the relevant cone structure is evidently given by the family of future directed causal cones, that is to say the cones made up by timelike and lightlike vectors $v$, i.e. 
with negative and vanishing Lorentzian norm respectively, satisfying, in addition, $h_{x}(X(x), v)<0$, for any timelike vector field $X$ providing the time orientation. They represent the physically admissible displacements pointing to the future.

Going back to our abstract setting, we need to introduce some definitions in order to present our results. The terminology is borrowed from Lorentzian geometry and General Relativity, and some explanations on it will be given later on. The cone structure $\mathcal{C}$ is said continuous if it possesses such property with respect to the Hausdorff metric, in local coordinates, up to intersection of the cones with the unit closed Euclidean ball. We call $\mathcal{C}$-causal and $\mathcal{C}$-future directed the integral curves of $\mathcal{C}_{x}, \mathcal{\mathcal { C }}_{x}$, respectively, where $\mathcal{\mathcal { C }}_{x}$ is the interior of $\mathcal{C}_{x}$. We define an order relation $\succ$ on cone structures by declaring $\mathcal{C}^{\prime} \succ \mathcal{C}$ whenever $\mathcal{C}_{x} \backslash\{0\} \subset \mathcal{C}_{x}^{\prime}$, for every $x \in M$.

The cone structure $\mathcal{C}$ is said causal if it is continuous and there are no closed $\mathcal{C}$-causal curves; if, in addition, there is a causal cone structure $\mathcal{C}^{\prime}$ with $\mathcal{C}^{\prime} \succ \mathcal{C}$, then $\mathcal{C}$ is called stably causal. Finally we define $\mathcal{C}$ globally hyperbolic if it is stably causal and all causal curves connecting two given points lie in a compact (possibly empty) subset of $M$. We establish, see Theorem 1.2, that globally hyperbolic is actually a stable property.

A function with the property of being strictly increasing on any nonconstant $\mathcal{C}$-causal curve is called a global time function for $\mathcal{C}$. If, in addition, it takes any value of its range when the argument varies on an inextendible (with maximal domain of definition) $\mathcal{C}$-causal curve, then the time function is said to be Cauchy. Moreover, we will say that $f: M \rightarrow \mathbb{R}$ is a smooth or $\mathrm{C}^{\infty}$ global time function for the cone structure $\mathcal{C}$, if its $\mathrm{C}^{\infty}$, and

$$
\forall x \in M, \forall v \in \mathcal{C}_{x} \backslash\{0\}, d_{x} f(v)>0 .
$$

Our main results are the following:

Theorem 1.1. If $\mathcal{C}$ is a stably causal cone structure on $M$, then there exists a $\mathrm{C}^{\infty}$ global time function for $\mathcal{C}$.

For the existence of smooth Cauchy time functions, we essentially need the already mentioned stability property for the global hyperbolicity. We believe that this result is indeed of independent interest.

Theorem 1.2. If the cone structure $\mathcal{C}$ is globally hyperbolic then there exists some $\mathcal{C}^{\prime} \succ \mathcal{C}$ which is also globally hyperbolic.

Theorem 1.3. For any globally hyperbolic cone structure $\mathcal{C}$ on $M$ there exists a $\mathrm{C}^{\infty}$ Cauchy global time function $f$ for $\mathcal{C}$, i.e. a smooth global time function such that

$$
\lim _{t \rightarrow a} f(\gamma(t))=-\infty \quad \text { and } \quad \lim _{t \rightarrow b} f(\gamma(t))=+\infty
$$

for any inextendible $\mathcal{C}$-causal curve $\gamma:] a, b[\rightarrow M$. 
In the context of space-times and future directed causal cone structures the time functions are clearly related to the existence of foliations of the space by surfaces of simultaneity, which is a central problem in General Relativity. If $f$ is a global time function then all its levels $f^{-1}(c)$ are achronal in the sense that there cannot be any causal connection between different points of it. If, in addition, the function $f$ is Cauchy time function then any level is accordingly a Cauchy surface, which means that any inextendible causal curve intersects it exactly once. The name originates from the fact that it is the natural region to pose initial conditions for Einstein's equations.

The causality condition seems quite natural in this setting since the violation of it would apparently yield causality breakdown: one could travel into one's past. Notice also that this requirements rules out compact space-time since it can be proved, see [2] Proposition 3.10, that compactness and causality are incompatible. The global hyperbolicity ensures that no causal curve joining two points can reach the edge of the space-time, the term is related to some special properties holding for the wave equation in this case.

The paper of Geroch [9] is seminal in the topic. Geroch establishes there the equivalence of global hyperbolicity and existence of a topological Cauchy hypersurface $S$. The property that the space-time is homeomorphic to $\mathbb{R} \times S$ is, in addition, derived in the so-called Splitting Theorem. A modification of Geroch's technique allows also to show the stable causality being equivalent to the existence of a continuous global time function.

The possibility of going beyond the topological nature of these results and giving a differential version of them, has remained as an open problem for a long period and, after some fallacious attempts, has been only recently solved by Bernal and Sánchez, see [4]. Starting from Geroch's Splitting Theorem, these authors first prove the existence of a smooth Cauchy surface and from this they construct a smooth Cauchy time function.

We tackle the issue from a new angle and propose an approach which stays independent of Geroch and Bernal-Sánchez contributions, and use instead some tools and results issued from weak KAM theory, see [8] for a comprehensive treatment of this topic. This connection is, at a first sight, quite surprising since such theory concerns the qualitative analysis of Hamilton-Jacobi equations at the critical value, i.e. at the minimum level for which a.e. subsolutions do exist, while, in the time function problem, no Hamiltonians are involved.

To bridge the gap, we first notice that what really matters in the study of a stationary Hamilton-Jacobi equations of the form $H(x, D u)=a$ is not the whole Hamiltonian but just the $a$-sublevels of it, namely the sets

$$
\{p \mid H(x, p) \leq a\}
$$

that we assume in this discussion with non-empty interior. Under the customary conditions of continuity, convexity and coercivity on $H$, the map associating to any $x$ the corresponding $a$-sublevel is convex compact valued and continuous 
with respect to the Hausdorff metric. These are the same kind of conditions that we have required for the map $x \mapsto \mathcal{C}_{x}$, and also hold true for $x \mapsto \mathcal{C}_{x}^{*}$, where $\mathcal{C}_{x}^{*}$ is the polar cone of $\mathcal{C}_{x}$, apart the compactness that we recover through suitable truncations.

The property of being a strict subsolution to the equation for a smooth function can be expressed by requiring its differential to be contained in the interior of the $a$-sublevel for any $x$. In the same way a global smooth time function $f$ for $\mathcal{C}$ is characterized by the fact that it is solution of the partial differential inclusion $-D f(x) \in \mathcal{C}_{x}^{*}$. Similar ideas have already been used in [12] to construct a Lyapunov function for a multivalued dynamics enjoying suitable stability and attractiveness conditions.

In the Hamilton-Jacobi framework a smooth strict subsolution is constructed, through a partition of unity technique and mollification process, see [7], provided that for any point one can find a neighborhood $U$ of $x, \epsilon>0$, and an a.e. subsolution $u$ to the equation in the whole space, which, in addition satisfies the strict inequality $H(x, D u(x)) \leq a-\epsilon$ for a.e. $x \in U$. The obstruction to this procedure is then represented by the points around which no a.e. global subsolution is strict. This set is named after Aubry and a crucial step in the analysis is to provide a metric characterization of it. This is made through the introduction of an intrinsic length for curves which again only depends on the $a$-sublevels of the Hamiltonian, and, more precisely, on their support functions, see [7]. In our case the intrinsic length is defined starting from the cone structure $\mathcal{C}$, and in a sense it indicates the deviation of a curve from being $\mathcal{C}$-causal, see (15), (20), so that a nonconstant trajectory possess zero intrinsic length if and only if it is $\mathcal{C}$-causal

The property detecting the points of the Aubry set is thus given via comparison of this intrinsic length and the natural length induced in the ground space by some reference Riemannian metric. More precisely a point belongs to the Aubry set if and only if there is a sequence of closed curves passing through it with Riemannian length bounded from below by a positive constant and arbitrarily small intrinsic length. The role of closed curves in both of definitions of Aubry set and causal cone structure, and the fact that the intrinsic length is zero whenever $\gamma$ is causal, cast some light on the way where weak KAM theory enters in our topic.

The crucial point is to show that, given $\mathcal{C}^{\prime} \succ \mathcal{C}$ the reference metric can be chosen in such a way that if the intrinsic length related to $\mathcal{C}$ of a curve joining two points $x$ and $y$ is small compared to the natural one then $x$ can be also connected to $y$ through a $\mathcal{C}^{\prime}$-future directed curve, see Lemma 2.7, Proposition 2.24, Theorem 3.4. If, in addition, $\mathcal{C}$ is stably causal and we pick $\mathcal{C}^{\prime}$ causal, this forces $x \neq y$, which shows that no cycle can have $\mathcal{C}$-length small compared to the natural one, and so, under the stable causality assumption, the corresponding Aubry set is empty, which yields Theorem 1.1.

Theorem 1.2 is essentially based on some stability properties holding in the class of $\mathcal{C}$-causal curves, and is, in turn, crucial for proving Theorem 1.3. Here 
we also exploit a density property for smooth global time functions, see Theorem 5.1 .

\section{Cone Structures}

\subsection{Cones in $\mathbb{R}^{N}$}

We consider a finite dimensional Euclidean space $\mathbb{R}^{N}$ and denote by $|\cdot|$ its Euclidean norm. For every $x \in \mathbb{R}^{N}$, we use the canonical identification of the tangent $T_{x} \mathbb{R}^{N}$ with $\mathbb{R}^{N}$. Given $v_{0}, v_{1}$ in $\mathbb{R}^{N}$ we will denote by $v_{0} \cdot v_{1}$ the usual Euclidean scalar product of $v_{0}$ and $v_{1}$. We systematically identify $\mathbb{R}^{N}$ with its dual $\left(\mathbb{R}^{N}\right)^{*}$, using the canonical basis on $\mathbb{R}^{N}$. Accordingly, if $p \in\left(\mathbb{R}^{N}\right)^{*}, v \in \mathbb{R}^{N}$, we will also use the scalar product notation, $p \cdot v$, to denote the action $p(v)$ of $p$ on $v$.

We write $\mathbb{B}$ for the closed unit Euclidean ball of $\mathbb{R}^{N}$ centered at 0 , accordingly $x+r \mathbb{B}$ will denote the closed ball of radius $r$ centered at $x$, for any $x \in \mathbb{R}^{N}$, $r>0$. For every closed non-empty set $K \subset \mathbb{R}^{N}$, the distance $d(x, K)$ of any $x$ from $K$ is defined by

$$
d(x, K)=\inf _{y \in K}|x-y|
$$

By the compactness of balls in the Euclidean space, this infimum is achieved. The points in $K$ realizing such infimum are called projections of $x$ on $K$, and the set made up by them is denoted by $\operatorname{proj}_{K}(x)$. As is well-known, the projection is unique if $K$ is, in addition, convex, since the norm $|\cdot|$ is Euclidean. In fact, in that case, for $v \in \mathbb{R}^{N}$, the projection of $v$ on $K$ is the unique $v_{0} \in K$ such that

$$
\left(v-v_{0}\right) \cdot\left(w-v_{0}\right) \leq 0 \quad \text { for any } w \in K .
$$

In the case where $K$ is closed and convex, we will denote by $\operatorname{proj}_{K}(v)$ the unique $v_{0} \in K$ such that $d(v, K)=d\left(v, v_{0}\right)=\left|v-v_{0}\right|$. It follows from property (1) that for a closed and convex subset $K \subset \mathbb{R}^{N}$ the map $\operatorname{proj}_{K}: \mathbb{R}^{N} \rightarrow K$ has Lipschitz constant equal to 1 , i. e.

$$
\left|\operatorname{proj}_{K}(v)-\operatorname{proj}_{K}\left(v^{\prime}\right)\right| \leq\left|v-v^{\prime}\right| \quad \text { for all } v, v^{\prime} \in \mathbb{R}^{N} .
$$

For an arbitrary non-empty closed set, still denoted by $K$, such that $\mathbb{R}^{N} \backslash K \neq \emptyset$ (or equivalently $\partial K \neq \emptyset$ ), it is convenient to introduce the signed distance given by the formula

$$
d^{\#}(\cdot, K)=2 d(\cdot, K)-d(\cdot, \partial K) .
$$

Proposition 2.1. If $K$ is a closed non-empty subset of $\mathbb{R}^{N}$ with $\mathbb{R}^{N} \backslash K \neq \emptyset$, we have

$$
d^{\#}(v, K)= \begin{cases}d(v, K)=d(v, \partial K) & \text { if } v \notin \stackrel{\circ}{K}, \\ -d(v, \partial K) & \text { if } v \in K .\end{cases}
$$

In particular, we have $\left|d^{\#}(v, K)\right|=d(v, \partial K)$, for every $v \in \mathbb{R}^{N}$. 
Proof. Given the definition of $d^{\#}$, it suffices to show that $d(v, K)=d(v, \partial K)$, if $v \notin \stackrel{\circ}{K}$. We obviously have $d(v, K) \leq d(v, \partial K)$. Moreover, if $v_{0} \in K$ is such that $d\left(v, v_{0}\right)=d(v, K)$, then, by connectedness, the segment $\left[v, v_{0}\right]$ must intersect $\partial K$ in a point $v_{1}$. Using $d\left(v, v_{0}\right)=d(v, K)$, we must have $v_{1}=v_{0}$, and $d(v, \partial K) \leq d\left(v, v_{0}\right)=d(v, K)$.

Another useful result is:

Proposition 2.2. If $K$ is a closed convex subset of $\mathbb{R}^{N}$ with $\mathbb{R}^{N} \backslash K \neq \emptyset$, then both the distance and the signed distance are convex functions on $\mathbb{R}^{N}$

In fact, it is well-known that $d(\cdot, K)$ is convex when $K$ is convex. For the signed distance this is not as well known, but still not difficult to prove. Anyway, we will use this lemma for closed convex cones with vertex at 0 (called just cones later), and this case follows from Lemma 2.4 below, where, in particular, it is, shown that both the distance and the signed distancee to such a cone are a supremum of linear functions, hence they are convex.

It is useful to consider positively homogeneous subset of $\mathbb{R}^{N}$. A subset $C \subset \mathbb{R}^{N}$ is called positively homogeneous if

$$
\forall v \in C, \forall \lambda \geq 0, \lambda v \in C .
$$

For such a positively homogeneous subset $C$, it is clear that its closure $\bar{C}$ and its boundary $\partial C$ are homogeneous.

The next proposition summarizes the main properties of the distance to a positively homogeneous closed subset or a cone.

Proposition 2.3. Let $C$ be a closed non-empty positively homogeneous subset of $\mathbb{R}^{N}$. If $v \in \mathbb{R}^{N}, \lambda \geq 0$, and $v_{0} \in C$ is a projection of $v$ on $C$, i.e. $d\left(v, v_{0}\right)=d(v, C)$ then we have

(i) $d(\lambda v, C)=\lambda d(v, C)$;

(ii) $\lambda v_{0}$ is a projection of $\lambda v$ on $C$;

(iii) $\left(v-v_{0}\right) \cdot v_{0}=0$;

(iv) $\left|v_{0}\right| \leq|v|$;

(v) if $v \in \mathbb{B}$ then $d(v, C)=d(v, C \cap \mathbb{B})$;

(vi) if $v \in \partial \mathbb{B}$, then $d(v, C \cap \partial \mathbb{B}) \leq 2 d(v, C \cap \mathbb{B})=2 d(v, C)$.

Proof. Properties (i) and (ii) are immediate.

To prove (iii), we remark that the function $\varphi:\left[0,+\infty\left[\rightarrow\left|v-t v_{0}\right|^{2}\right.\right.$ has a minimum a $t=1$. Hence $\varphi^{\prime}(1)=0$. But $\varphi^{\prime}(1)=-2\left(v-v_{0}\right) \cdot v_{0}$.

To prove (iv), we note that the triangle with vertices at $0, v_{0}$ and $v$ has a right angle at $v_{0}$. Therefore $|v|^{2}=\left|v-v_{0}\right|^{2}+\left|v_{0}\right|^{2}$, and $\left|v_{0}\right| \leq|v|$. 
To prove (v), using $C \cap \mathbb{B} \subset C$, we first notice that $d(v, C) \leq d(v, C \cap \mathbb{B})$. On the other hand, since $v \in \mathbb{B}$, the projection $v_{0}$ has norm $\leq 1$ by (iv). Therefore $v_{0} \in C \cap \mathbb{B}$. Hence

$$
d(v, C)=\left|v-v_{0}\right| \geq d(v, C \cap \mathbb{B}) .
$$

Consider now $v \in \partial \mathbb{B}$, and $v_{0}$ a projection of $v$ on $C$. If $v_{0}=0$, then $d(v, C)=$ $|v|=1$. Since the diameter of $\partial \mathbb{B}$ is equal to 2 , it follows (vi) holds if $v_{0}=0$. If $v_{0} \neq 0$, then we can consider $v_{0} /\left|v_{0}\right| \in \partial \mathbb{B}$. The triangle with vertices at $v, v_{0}$, and $v_{0} /\left|v_{0}\right|$ has a right angle at $v_{0}$. Hence

$$
\begin{aligned}
d(v, C \cap \partial \mathbb{B})^{2} & \leq\left|v-\frac{v_{0}}{\left|v_{0}\right|}\right|^{2} \\
& =\left|v-v_{0}\right|^{2}+\left|v_{0}-\frac{v_{0}}{\left|v_{0}\right|}\right|^{2} \\
& =\left|v-v_{0}\right|^{2}+\left(1-\left|v_{0}\right|\right)^{2} .
\end{aligned}
$$

Using (iv), and the triangle inequality, we note that $0 \leq|v|-\left|v_{0}\right| \leq\left|v-v_{0}\right|$. Since $|v|=1$, squaring yields $\left(1-\left|v_{0}\right|\right)^{2} \leq\left|v-v_{0}\right|^{2}$. Combining with the estimate above, we obtain $d(v, C \cap \partial \mathbb{B})^{2} \leq 2\left|v-v_{0}\right|^{2}=2 d(v, C)^{2}$. Using (v), this proves (vi).

Throughout the paper, we will simply say cone to mean a closed convex cone not containing any complete affine line, with vertex at 0 , and non-empty interior. These cones are usually qualified as complete, see [5], in the literature. We will denote the set of such cones by Cones $\left(\mathbb{R}^{N}\right)$.

Obviously a cone $\mathcal{C}^{0}$ is positively homogeneous, since we assume that its vertex is 0 . We can therefore apply to it Proposition 2.3. In particular, part (iii) of Proposition 2.3 and property (1) yield

$$
\left(v-\operatorname{proj}_{\mathcal{C}^{0}}(v)\right) \cdot w \leq 0 \quad \text { for any } w \in \mathcal{C}^{0} .
$$

For a cone $\mathcal{C}^{0} \subset \mathbb{R}^{N}$, we define its polar by

$$
\mathcal{C}^{0^{*}}=\left\{p \in\left(\mathbb{R}^{N}\right)^{*} \approx \mathbb{R}^{N} \mid p \cdot v \leq 0 \text { for } v \in \mathcal{C}^{0}\right\} .
$$

The polar $\mathcal{C}^{0^{*}}$ is also a (complete) cone, and is therefore in $\operatorname{Cones}\left(\left(\mathbb{R}^{N}\right)^{*}\right)=$ Cones $\left(\mathbb{R}^{N}\right)$. Note that if $\mathcal{C}^{0} \subset \mathcal{C}^{1}$ then $\mathcal{C}^{1^{*}} \subset \mathcal{C}^{0^{*}}$. Moreover, we have $\left(\mathcal{C}^{0}\right)^{* *}=\mathcal{C}^{0}$ by the Hahn-Banach Theorem.

It is convenient to give a dual characterization of the distance $d\left(\cdot, \mathcal{C}^{0}\right)$ and the signed distance $d^{\#}\left(\cdot, \mathcal{C}^{0}\right)$ from a cone. Note that the convexity of these functions is an immediate consequence of this characterization. 
Lemma 2.4. Let $\mathcal{C}^{0}$ be a cone, then for any $v \in \mathbb{R}^{N}$

$$
\begin{aligned}
d\left(v, \mathcal{C}^{0}\right) & =\max \left\{p \cdot v \mid p \in \mathcal{C}^{0^{*}} \cap \mathbb{B}\right\}, \\
d^{\#}\left(v, \mathcal{C}^{0}\right) & =\max \left\{p \cdot v \mid p \in \mathcal{C}^{0^{*}} \cap \partial \mathbb{B}\right\} .
\end{aligned}
$$

Proof. To simplify notations, we set for $v \in \mathbb{R}^{N}$

$$
\begin{aligned}
& \sigma(v)=\max \left\{p \cdot v \mid p \in \mathcal{C}^{0^{*}} \cap \mathbb{B}\right\} \\
& \hat{\sigma}(v)=\max \left\{p \cdot v \mid p \in \mathcal{C}^{0^{*}} \cap \partial \mathbb{B}\right\} .
\end{aligned}
$$

Note that $\sigma \geq 0$, since $0 \in\left(\mathcal{C}^{0}\right)^{*} \cap \mathbb{B}$. Obviously $\hat{\sigma} \leq \sigma$, and both $\sigma$ and $\hat{\sigma}$ are Lipschitz with Lipschitz constant 1 , as supremums of families of Lipschitz functions with Lipschitz constant 1.

Let us start by (4). Pick $v \in \mathbb{R}^{N}$, if $\sigma(v)=0$ then $v \in\left(\mathcal{C}^{0}\right)^{* *}=\mathcal{C}^{0}$ and so $d\left(v, \mathcal{C}^{0}\right)=0$. If instead $\sigma(v)>0$ then $\sigma(v)=p \cdot v$ with $p \in\left(\mathcal{C}^{0}\right)^{*} \cap \partial \mathbb{B}$, thus $\sigma(v)=\hat{\sigma}(v)$. Moreover we have

$$
\begin{aligned}
\sigma(v) & =p \cdot\left(v-\operatorname{proj}_{\mathcal{C}^{0}}(v)\right)+p \cdot \operatorname{proj}_{\mathcal{C}^{0}}(v) \\
& \leq p \cdot\left(v-\operatorname{proj}_{\mathcal{C}^{0}}(v)\right) \\
& \leq\left|v-\operatorname{proj}_{\mathcal{C}^{0}}(v)\right| \\
& =d\left(v, \mathcal{C}^{0}\right) .
\end{aligned}
$$

To prove the converse inequality we first derive from property (3) above that $\left(v-\operatorname{proj}_{\mathcal{C}^{0}}(v)\right) \cdot w \leq 0$, for any $w \in \mathcal{C}^{0}$. It follows that $v-\operatorname{proj}_{\mathcal{C}^{0}}(v) \in \mathcal{C}^{0^{*}}$. Consequently taking into account part (iii) of Proposition 2.3, we obtain

$$
\begin{aligned}
\sigma(v) & \geq\left(\frac{v-\operatorname{proj}_{\mathcal{C}^{0}}(v)}{\left|v-\operatorname{proj}_{\mathcal{C}^{0}}(v)\right|}\right) \cdot v \\
& =\left(\frac{v-\operatorname{proj}_{\mathcal{C}^{0}}(v)}{\left|v-\operatorname{proj}_{\mathcal{C}^{0}}(v)\right|}\right) \cdot\left(v-\operatorname{proj}_{\mathcal{C}^{0}}(v)\right) \\
& =d\left(v, \mathcal{C}^{0}\right) .
\end{aligned}
$$

Note that if $v \notin \mathcal{C}^{0}=\left(\mathcal{C}^{0}\right)^{* *}$ then $\sigma(v)>0$, and $\sigma(v)=\hat{\sigma}(v)$, as we have already pointed out in the first part of the proof. Summing up we have not only proved (4) but also that

$$
\sigma(v)=\hat{\sigma}(v)=d\left(v, \mathcal{C}^{0}\right)=d^{\#}\left(v, \mathcal{C}^{0}\right) \quad \text { for } v \notin \mathcal{C}^{0} .
$$

Since all involved function are continuous, the equality also holds on $\partial \mathcal{C}^{0}$. It follows that $(5)$ is true outside of $\mathcal{C}^{0}$.

It remains to prove $(5)$, for $v \in \stackrel{\mathcal{C}}{0}^{0}$. To simplify notation set $r_{0}=d\left(v, \partial \mathcal{C}^{0}\right)$. Since the closed Euclidean ball centered at $v$ with radius $r_{0}$ is contained in $\mathcal{C}^{0}$, we have $p \cdot\left(v-r_{0} w\right) \leq 0$ for every $p \in\left(\mathcal{C}^{0}\right)^{*} \cap \partial \mathbb{B}$ and every $w \in \mathbb{B}$. Hence, we 
obtain $p \cdot v \leq r_{0}(p \cdot w)$. If we now take the infimum of all $w \in \mathbb{B}$, taking into account that $|p|=1$, we obtain $p \cdot v \leq-r_{0}$, and finally $\hat{\sigma}(v) \leq-r_{0}=d^{\#}\left(v, \mathcal{C}^{0}\right)$.

To get the converse inequality, we pick a projection $v_{0}$ of $v$ on $\partial \mathcal{C}^{0}$. By what we showed above $\hat{\sigma}\left(v_{0}\right)=d^{\#}\left(v_{0}, \mathcal{C}^{0}\right)=0$ Since we know that $\hat{\sigma}$ has Lipschitz constant 1 , we obtain

$$
-\hat{\sigma}(v)=\hat{\sigma}\left(v_{0}\right)-\hat{\sigma}(v) \leq\left|v-v_{0}\right|=r_{0} .
$$

Hence $\hat{\sigma}(v) \geq-r_{0}=d^{\#}\left(v, \mathcal{C}^{0}\right)$.

We now come to the order $\prec$ on cones already given in the Introduction.

Definition 2.5. If $\mathcal{C}^{1}, \mathcal{C}^{2}$ are two cones in $\mathbb{R}^{N}$, we say that $\mathcal{C}^{1} \prec \mathcal{C}^{2}$ if $\mathcal{C}^{1} \backslash\{0\} \subset \mathcal{C}^{2}$.

Proposition 2.6. Let $\mathcal{C}^{1}, \mathcal{C}^{2}$ be two cones in $\mathbb{R}^{N}$. We have $\mathcal{C}^{1} \prec \mathcal{C}^{2}$ if and only if $\mathcal{C}^{2^{*}} \prec \mathcal{C}^{1^{*}}$

Proof. Since $\mathcal{C}^{* *}=\mathcal{C}$, we need only to prove the implication from left to right. We know that the compact set $\partial \mathbb{B} \cap \mathcal{C}^{1}$ is contained in $\mathcal{C}^{2}$. therefore we can find $\delta>0$ such that for every $v \in \partial \mathbb{B} \cap \mathcal{C}^{1}$ the Euclidean ball $v+\delta \mathbb{B} \subset \mathcal{C}^{2}$. If $p \in \mathcal{C}^{2^{*}}$, we then have

$$
p \cdot\left(v+v^{\prime}\right) \leq 0 \quad \text { for all } v \in \partial \mathbb{B} \cap \mathcal{C}^{1}, v^{\prime} \in \delta \mathbb{B} .
$$

We fix $v$ and take into account that $\sup \left\{p \cdot v^{\prime} \mid v^{\prime} \in \delta \mathbb{B}\right\}=\delta|p|$, to obtain

$$
p \cdot v+\delta|p| \leq 0 \quad \text { for any } v \in \partial \mathbb{B} \cap \mathcal{C}^{1} .
$$

In particular we get

$$
\left(p+p^{\prime}\right) \cdot v \leq p \cdot v+\left|p^{\prime}\right||v| \leq p \cdot v+\delta|p| \leq 0 \quad \text { for any } p^{\prime} \in \delta|p| \mathbb{B}, v \in \partial \mathbb{B} \cap \mathcal{C}^{1}
$$

Therefore by homogeneity $p+\delta|p| \mathbb{B} \subset \mathcal{C}^{1^{*}}$, for every $p \in \mathcal{C}^{2^{*}}$, which implies $\mathcal{C}^{2^{*}} \backslash\{0\} \subset \mathcal{C}^{1^{*}}$

We end this subsection on cones with a Lemma that will be used later. As usual, we denote by $\ell(\gamma)$ the Euclidean length of a curve $\gamma$ contained in $\mathbb{R}^{N}$.

Lemma 2.7. Let $\mathcal{C}^{1}, \mathcal{C}^{2}$ be two cones in $\mathbb{R}^{N}$ with $\mathcal{C}^{1} \prec \mathcal{C}^{2}$. There exists $\delta>0$ such that for any Lipschitz curve $\gamma:[a, b] \rightarrow \mathbb{R}^{N}$, with

$$
\int_{a}^{b} d\left(\dot{\gamma}(s), \mathcal{C}^{1}\right) d s<\delta \ell(\gamma)
$$

one has $\gamma(b)-\gamma(a) \in \mathcal{C}^{2}$. 
Proof. Note that (6) implies that $\ell(\gamma)>0$, hence $\gamma$ is not constant. By Proposition 2.6 above the compact subset $S=\partial \mathbb{B} \cap \mathcal{C}^{2^{*}}$ is contained in the interior of $\mathcal{C}^{1^{*}}$, we can thus find $\epsilon>0$ such that $p+\epsilon \mathbb{B} \subset \mathcal{C}^{1^{*}}$, for any $p \in S$. This implies, in view of (4)

$$
d\left(v, \mathcal{C}^{1}\right) \geq \frac{p+p^{\prime}}{1+\epsilon} \cdot v \quad \text { for any } v \in \mathbb{R}^{N}, p \in S, p^{\prime} \in \epsilon \mathbb{B} .
$$

Multiplying by $1+\epsilon$, and taking the supremum over $p^{\prime} \in \epsilon \mathbb{B}$ yields

$$
(1+\epsilon) d\left(v, \mathcal{C}^{1}\right) \geq \epsilon|v|+p \cdot v \quad \text { for any } v \in \mathbb{R}^{N}, p \in S
$$

and consequently

$$
(1+\epsilon) \int_{a}^{b} d\left(\dot{\gamma}(s), \mathcal{C}^{1}\right) d s \geq 2 \epsilon \ell(\gamma)+p(\gamma(b)-\gamma(a))
$$

We choose now $\delta>0$ such that $(1+\epsilon) \delta<\epsilon$. If $(6)$ holds for such a $\delta$, we therefore get

$$
p \cdot(\gamma(b)-\gamma(a))<[(1+\epsilon) \delta-\epsilon] \ell(\gamma)<0, \text { for any } p \in S,
$$

which gives $\gamma(b)-\gamma(a) \in \stackrel{\circ}{\mathcal{C}}^{2}$.

\subsection{Metric structure on the set of cones in $\mathbb{R}^{N}$}

We will use the Hausdorff metric on compact sets to define a distance in $\operatorname{Cones}\left(\mathbb{R}^{N}\right)$.

Before doing that, we first recall some basic facts about Hausdorff distance. If $(X, d)$ is a metric space, one defines the Hausdorff distance $d^{H}$ between compact subsets of $X$ by

$$
d^{H}\left(K_{1}, K_{2}\right)=\max \left\{\max _{x \in K_{1}} d\left(x, K_{2}\right), \max _{y \in K_{2}} d\left(y, K_{1}\right)\right\} .
$$

The fact that $d^{H}$ is a metric follows from the following useful identity

$$
d^{H}\left(K_{1}, K_{2}\right)=\sup _{x \in X}\left|d\left(x, K_{1}\right)-d\left(x, K_{2}\right)\right|
$$

Suppose that $f: X \rightarrow Y$ is a Lipschitz map from $(X, d)$ to the metric space $(Y, d)$, with Lipschitz constant $L$. It is not difficult to verify that for any pair $K_{1}, K_{2}$ of closed subsets in $X$ we have

$$
d^{H}\left(f\left(K_{1}\right), f\left(K_{2}\right)\right) \leq L d^{H}\left(K_{1}, K_{2}\right) .
$$

We will denote by $\mathcal{K}(X)$ the set of non-empty compact subsets of $X$. We will always tacitly consider that $\mathcal{K}(X)$ is a metric space with $d^{H}$ as a metric.

The following results will be useful at some later point. 
Lemma 2.8. For $x, x \in X$, and $K, K^{\prime} \in \mathcal{K}(X)$, we have

$$
\left|d(x, K)-d\left(x^{\prime}, K^{\prime}\right)\right| \leq d\left(x, x^{\prime}\right)+d^{H}\left(K, K^{\prime}\right)
$$

Therefore the map $X \times \mathcal{K}(X),(x, K) \mapsto d(x, K)$ is Lipschitz.

Proof. The map $x \mapsto d(x, K)$ has Lipschitz constant 1 , therefore

$$
\begin{aligned}
\left|d(x, K)-d\left(x^{\prime}, K^{\prime}\right)\right| & \leq\left|d(x, K)-d\left(x^{\prime}, K\right)\right|+\left|d\left(x^{\prime}, K\right)-d\left(x^{\prime}, K^{\prime}\right)\right| \\
& \leq d\left(x, x^{\prime}\right)+d^{H}\left(K, K^{\prime}\right),
\end{aligned}
$$

where the last inequality was obtained using also (8) above.

We will need to consider Lipschitz functions on the product of two metric spaces $(X, d)$ and $(\Lambda, d)$. Note that all the metrics that one usually puts on $X \times \Lambda$ are of the form $d\left(\left(x_{1}, \lambda_{1}\right),\left(x_{2}, \lambda_{2}\right)\right)=\left\|\left(d\left(x_{1}, x_{2}\right), d\left(\lambda_{1}, \lambda_{2}\right)\right)\right\|$, where $\|\cdot\|$ is a norm on $\mathbb{R}^{2}$. All these metrics define the product topology, and they are all Lipschitz equivalent (like norms on $\mathbb{R}^{2}$ ). Therefore it makes perfect sense to speak of Lipschitz or locally Lipschitz functions on the product of two spaces We will, for example, assume that the metric on $X \times \Lambda$ is given by

$$
d\left(\left(x_{1}, \lambda_{1}\right),\left(x_{2}, \lambda_{2}\right)\right)=\max \left[d\left(x_{1}, x_{2}\right), d\left(\lambda_{1}, \lambda_{2}\right)\right] .
$$

Lemma 2.9. Suppose that $X, \Lambda$ are metric spaces, and that $f: X \times \Lambda \rightarrow \mathbb{R}$ is a continuous function. Let $\mathcal{K}(X)$ denote the set of non-empty compact subsets of $X$ endowed with the Hausdorff distance $d^{H}$. Then the two functions $f_{+}, f_{-}$: $\mathcal{K}(X) \times \Lambda \rightarrow \mathbb{R}$ defined by

$$
f_{+}(K, \lambda)=\sup _{x \in K} f(x, \lambda) \text { and } f_{-}(K, \lambda)=\inf _{x \in K} f(x, \lambda)
$$

are continuous. Moreover, if $f$ is Lipschitz (resp. locally Lipschitz), so are $f_{+}$ and $f_{-}$.

Proof. We will deal with the case of $f_{+}$. Fix $C_{0} \in \mathcal{K}(X)$ and $\lambda_{0} \in \Lambda$. We define $\rho:[0,+\infty[\rightarrow[0,+\infty[$ by

$$
\rho(\delta)=\sup \left|f(x, \lambda)-f\left(y, \lambda_{0}\right)\right|
$$

where the sup is taken over all $x, y \in X$, and $\lambda \in \Lambda$ satisfying $d\left(x, C_{0}\right) \leq$ $\delta, d\left(y, C_{0}\right) \leq \delta, d(x, y) \leq \delta$, and $d\left(\lambda, \lambda_{0}\right) \leq \delta$. Since $C_{0}$ is compact, and $f$ is assumed continuous, one can show (for example by contradiction) that $\rho(\delta) \rightarrow 0$, when $\delta \rightarrow 0$. To prove the continuity of $f_{+}$at $\left(C_{0}, \lambda_{0}\right)$ it suffices to show that

$$
\left|f_{+}(C, \lambda)-f_{+}\left(C_{0}, \lambda_{0}\right)\right| \leq \rho\left[\max \left(d^{H}\left(C, C_{0}\right), d\left(\lambda, \lambda_{0}\right)\right)\right] .
$$


Let us set $\delta=\max \left(d^{H}\left(C, C_{0}\right), d\left(\lambda, \lambda_{0}\right)\right)$. For $x \in C$, we can find $y \in C_{0}$ such that $d(x, y) \leq \delta$. Since $d\left(x, C_{0}\right) \leq \delta, d\left(y, C_{0}\right) \leq \delta, d(x, y) \leq \delta, d\left(\lambda, \lambda_{0}\right) \leq \delta$, we have

$$
\left|f(x, \lambda)-f\left(y, \lambda_{0}\right)\right| \leq \rho(\delta) .
$$

Using $y \in C_{0}$, this yields

$$
f(x, \lambda) \leq f\left(y, \lambda_{0}\right)+\rho(\delta) \leq f_{+}\left(C_{0}, \lambda_{0}\right)+\rho(\delta) .
$$

Taking the sup over all $x \in C$, we get

$$
f_{+}(C, \lambda)-f_{+}\left(C_{0}, \lambda_{0}\right) \leq \rho(\delta) .
$$

Conversely if $x \in C_{0}$, we can find $y \in C$, with $d(x, y) \leq \delta$. By the same argument as above

$$
f\left(x, \lambda_{0}\right) \leq f(y, \lambda)+\rho(\delta) \leq f_{+}(C, \lambda)+\rho(\delta),
$$

and

$$
f_{+}\left(C_{0}, \lambda_{0}\right)-f_{+}(C, \lambda) \leq \rho(\delta) .
$$

It remains to deal with the Lipschitz and locally Lipschitz cases. The Lipschitz case is obtained from the proof above because we can then take $\rho(\delta)=K \delta$, where $K$ is a Lipschitz constant for $f$.

We will now treat the locally Lipschitz case. Fix $C_{0} \in \mathcal{K}(X)$ and $\lambda_{0} \in \Lambda$. By definition of locally Lipschitz, for each $x \in X$, we can find $\delta_{x}>0$, and $L_{x}<+\infty$, such that $f$ is Lipschitz on $B\left(x, 2 \delta_{x}\right) \times B\left(\lambda_{0}, \delta_{x}\right)$ with Lipschitz constant $\leq L_{x}$. Note that $f$ is therefore bounded on $B\left(x, 2 \delta_{x}\right) \times B\left(\lambda_{0}, \delta_{x}\right)$.

By compactness of $C_{0}$, we can find a finite number $x_{1}, \ldots, x_{n}$ of points in $C_{0}$ such that $C_{0} \subset \cup_{i=1}^{n} B\left(x_{i}, \delta_{x_{i}}\right)$. Setting $\delta=\min _{i=1}^{n} \delta_{x_{i}}>0$, and $L_{1}=\max _{i=1}^{n} L_{x_{i}}$, it can be checked that for $x_{1}, x_{2} \in B_{\delta}(C)=\{z \in X \mid d(z, C)<\delta\}$, with $d\left(x_{1}, X_{2}\right)<\delta$, and $\lambda_{1}, \lambda_{2} \in B\left(\lambda_{0}, \delta\right)$, we have

$$
\left|f\left(x_{1}, \lambda_{1}\right)-f\left(x_{2}, \lambda_{2}\right)\right| \leq L \max \left[d\left(x_{1}, x_{2}\right), d\left(\lambda_{1}, \lambda_{2}\right)\right] .
$$

Note also that $B_{\delta}\left(C_{0}\right) \times B\left(\lambda_{0}, \delta\right) \subset \cup_{i=1}^{n} B\left(x_{i}, 3 \delta_{x_{i}}\right) \times B\left(\lambda_{0}, \delta_{x_{i}}\right)$, and that $f$ is bounded on each element of that finite union. Therefore $f$ is bounded on $B_{\delta}\left(C_{0}\right) \times$ $B\left(\lambda_{0}, \delta\right)$. If $M$ is such a bound of $f$, and $L=\max \left(L_{1}, 2 M / \delta\right)$, it is easy to check that $f$ is Lipschitz on $B_{\delta}\left(C_{0}\right) \times B\left(\lambda_{0}, \delta\right)$, with Lipschitz constant $L$. Since any $C \in \mathcal{K}(X)$ with $d^{H}\left(C, C_{0}\right)<\delta$ satisfies $C \subset B_{\delta}\left(C_{0}\right)$. From the Lipschitz case applied to $B_{\delta}\left(C_{0}\right)$ instead of $X$, and $B\left(\lambda_{0}, \delta\right)$ instead of $\Lambda$, we obtain that $f_{+}$is Lipschitz on $\left\{C \in \mathcal{K}(X) \mid d^{H}\left(C, C_{0}\right)<\delta\right\} \times B\left(\lambda_{0}, \delta\right)$.

Lemma 2.10. Let $\Lambda$ be a metric space, and $C$ a compact convex subset of some Euclidean space $\mathbb{R}^{N}$. Suppose $f: C \times \Lambda \rightarrow \mathbb{R}$ is a continuous function such that, for every $\lambda \in \Lambda$, the function $x \mapsto f(x, \lambda)$ is convex and continuous on $C$.

For $\lambda \in \Lambda$, we set

$$
C_{\lambda}=\{x \in C \mid f(x, \lambda) \leq 0\}
$$


Then the set $\Lambda_{f}=\left\{\lambda \in \Lambda \mid \inf _{x \in C} f(x, \lambda)<0\right\}$ is open and the map $\Lambda_{f} \rightarrow$ $\mathcal{K}(C), \lambda \mapsto C_{\lambda}$ is continuous. Moreover, if $f$ is locally Lipschitz, then the map $\lambda \mapsto C_{\lambda}$ is locally Lipschitz on the open subset $\Lambda_{f}$.

Proof. Define $F: \Lambda \times \Lambda \rightarrow[0,+\infty[$ by

$$
F\left(\lambda_{1}, \lambda_{2}\right)=\sup _{x \in C}\left|f\left(x, \lambda_{1}\right)-f\left(x, \lambda_{2}\right)\right|
$$

Note that $F$ is finite everywhere by the compactness of $C$ and the continuity of $f$. Moreover, the same continuity and compactness implies that for $\lambda_{1}$ fixed, we have

$$
\lim _{\lambda_{2} \rightarrow \lambda_{1}} F\left(\lambda_{1}, \lambda_{2}\right)=0 .
$$

Suppose now that $\lambda_{0} \in \Lambda_{f}$. We can find $x_{0} \in C$ such that $f\left(x_{0}, \lambda_{0}\right)<0$. Therefore by continuity of $\lambda \mapsto f\left(\lambda, x_{0}\right)$ we can find a neighborhood $V$ of $\lambda_{0}$ and $\epsilon>0$ such that

$$
\forall \lambda \in V, f\left(x_{0}, \lambda\right) \leq-\epsilon
$$

Set $K=\operatorname{diam}(C) / \epsilon$. We will first show that

$$
\forall \lambda_{1}, \lambda_{2} \in V, \forall x \in C_{\lambda_{1}}, d\left(x, C_{\lambda_{2}}\right) \leq K F\left(\lambda_{1}, \lambda_{2}\right) .
$$

We fix $\lambda_{1}, \lambda_{2} \in V$, and $x \in C_{\lambda_{1}}$. Since $f\left(x, \lambda_{1}\right) \leq 0$, we have

$$
f\left(x, \lambda_{2}\right) \leq f\left(x, \lambda_{2}\right)-f\left(x, \lambda_{1}\right) \leq F\left(\lambda_{1}, \lambda_{2}\right) .
$$

If $x \in C_{\lambda_{2}}$, then $d\left(x, C_{\lambda_{2}}\right)=0$ and (10) is clear, since $F$ is non-negative. Therefore we can assume $f\left(x, \lambda_{2}\right)>0$. We set $x_{\alpha}=(1-\alpha) x_{0}+\alpha x, \alpha \in[0,1]$. Since $f\left(x_{0}, \lambda_{2}\right)<0<f\left(x, \lambda_{2},\right)$, by continuity of $f$ in the first argument, we can find $\alpha_{0}$ such that ' $f\left(x_{\alpha_{0}}, \lambda_{2}\right)=0$. Using the convexity of $f\left(\cdot, \lambda_{2}\right.$, we obtain

$$
0=f\left(x_{\alpha_{0}}, \lambda_{2}\right) \leq\left(1-\alpha_{0}\right) f\left(x_{0}, \lambda_{2}\right)+\alpha_{0} f\left(x, \lambda_{2}\right) .
$$

Since $f\left(x_{0}, \lambda_{2}\right) \leq-\epsilon, f\left(x, \lambda_{2}\right) \leq F\left(\lambda_{1}, \lambda_{2}\right)$, and $F$ is non-negative, this implies

$$
0 \leq-\left(1-\alpha_{0}\right) \epsilon+\alpha_{0} F\left(\lambda_{1}, \lambda_{2}\right) \leq-\left(1-\alpha_{0}\right) \epsilon+F\left(\lambda_{1}, \lambda_{2}\right) .
$$

Therefore

$$
\left(1-\alpha_{0}\right) \leq \frac{F\left(\lambda_{1}, \lambda_{2}\right)}{\epsilon}
$$

Since $f\left(x_{\alpha_{0}}, \lambda_{2}\right)=0$, we get

$$
\begin{aligned}
d\left(x, C_{\lambda_{2}}\right) & \leq\left|x-x_{\alpha_{0}}\right|=\left(1-\alpha_{0}\right)\left|x-x_{0}\right| \\
& \leq \frac{F\left(\lambda_{1}, \lambda_{2}\right)}{\epsilon} \operatorname{diam}(C) \\
& =K F\left(\lambda_{1}, \lambda_{2}\right) .
\end{aligned}
$$


The inequality (10) implies

$$
\forall \lambda_{1}, \lambda_{2} \in V, \sup _{x \in C_{\lambda_{1}}} d\left(x, C_{\lambda_{2}}\right) \leq K F\left(\lambda_{1}, \lambda_{2}\right) .
$$

Exchanging the role of $\lambda_{1}$ and $\lambda_{2}$, we obtain

$$
\forall \lambda_{1}, \lambda_{2} \in V, d^{H}\left(C_{\lambda_{1}}, C_{\lambda_{2}}\right) \leq K F\left(\lambda_{1}, \lambda_{2}\right) .
$$

Together with (2.2), this proves the continuity of the map $\lambda \mapsto C_{\lambda}$ on the neighborhood $V$ of $\lambda_{0}$.

To prove the last part of the Lemma, suppose $f$ is locally Lipschitz. We fix $\lambda_{0} \in \Lambda$. As in the proof of the previous lemma, we could find $\delta>0$ and $L<+\infty$ such that $f$ is Lipschitz on $C \times B\left(\lambda_{0}, \delta\right)$ with Lipschitz constant $\leq L$.

$$
\forall \lambda_{1}, \lambda_{2} \in B\left(\lambda_{0}, \delta\right), F\left(\lambda_{1}, \lambda_{2}\right) \leq \operatorname{Ld}\left(\lambda_{1}, \lambda_{2}\right) .
$$

Hence, by what we have just obtained applied to $B\left(\lambda_{0}, \delta\right)$ instead of $\Lambda$, the map $\lambda \mapsto C_{\lambda}$ is Lipschitz on the neighborhood $B\left(\lambda_{0}, \delta\right)$ of $\lambda_{0}$.

We now proceed to define a distance $\delta^{H}$ on $\operatorname{Cones}\left(\mathbb{R}^{N}\right)$ by

$$
\delta^{H}\left(\mathcal{C}^{1}, \mathcal{C}^{2}\right)=d^{H}\left(\mathcal{C}^{1} \cap \mathbb{B}, \mathcal{C}^{2} \cap \mathbb{B}\right),
$$

for any pair $\mathcal{C}^{1}, \mathcal{C}^{2} \in \operatorname{Cones}\left(\mathbb{R}^{N}\right)$, see [3].

We will always consider Cones $\left(\mathbb{R}^{N}\right)$ as a metric space endowed with the metric $\delta^{H}$. From the next Lemma, $\delta^{H}$ is equivalent to the metric $\hat{\delta}^{H}$ defined by

$$
\hat{\delta}^{H}\left(\mathcal{C}^{1} \mathcal{C}^{2}\right)=d^{H}\left(\mathcal{C}^{1} \cap \partial \mathbb{B}, \mathcal{C}^{2} \cap \partial \mathbb{B}\right) .
$$

Lemma 2.11. For every $\mathcal{C}^{1}, \mathcal{C}^{2} \in \operatorname{Cones}\left(\mathbb{R}^{N}\right)$, we have

$$
\delta^{H}\left(\mathcal{C}^{1}, \mathcal{C}^{2}\right) \leq d^{H}\left(\mathcal{C}^{1} \cap \partial \mathbb{B}, \mathcal{C}^{2} \cap \partial \mathbb{B}\right) \leq 2 \delta^{H}\left(\mathcal{C}^{1}, \mathcal{C}^{2}\right) .
$$

Proof. If $v \in \mathcal{C}^{1} \cap \mathbb{B}$, and $v \neq 0$, taking into account Proposition 2.3 we get

$$
\begin{aligned}
d\left(v, \mathcal{C}^{2} \cap \mathbb{B}\right) & =d\left(v, \mathcal{C}^{2}\right)=|v| d\left(\frac{v}{|v|}, \mathcal{C}^{2}\right) \\
& \leq d\left(\frac{v}{|v|}, \mathcal{C}^{2}\right) \leq d\left(\frac{v}{|v|}, \mathcal{C}^{2} \cap \partial \mathbb{B}\right) \\
& \leq d^{H}\left(\mathcal{C}^{1} \cap \partial \mathbb{B}, \mathcal{C}^{2} \cap \partial \mathbb{B}\right),
\end{aligned}
$$

which yields

$$
\max _{v \in \mathcal{C}^{1} \cap \mathbb{B}} d\left(v, \mathcal{C}^{2} \cap \mathbb{B}\right) \leq d^{H}\left(\mathcal{C}^{1} \cap \partial \mathbb{B}, \mathcal{C}^{2} \cap \partial \mathbb{B}\right) .
$$

By symmetry, we get the left hand side inequality of the lemma. For the right hand side, we use the item (vi) in the statement of Proposition 2.3 to obtain for $v \in \mathcal{C}^{1} \cap \partial \mathbb{B}$

$$
d\left(v, \mathcal{C}^{2} \cap \partial \mathbb{B}\right) \leq 2 \delta\left(v, \mathcal{C}^{2} \cap \mathbb{B}\right) \leq 2 \delta^{H}\left(\mathcal{C}^{1}, \mathcal{C}^{2}\right) .
$$

Again by symmetry, this yields the right hand side inequality of the lemma. 
From Lemma 2.4, we first derive that the map $\mathcal{C}^{0} \mapsto \mathcal{C}^{0^{*}}$ is an isometry with respect to $\delta^{H}$.

Proposition 2.12. For every pair $\mathcal{C}^{1}, \mathcal{C}^{2} \in \operatorname{Cones}\left(\mathbb{R}^{N}\right)$, we have $\delta^{H}\left(\mathcal{C}^{1}, \mathcal{C}^{1}\right)=$ $\delta^{H}\left(\mathcal{C}^{1^{*}}, \mathcal{C}^{2^{*}}\right)$.

Proof. Taking into account Lemma 2.4, we have by duality

$$
d\left(p, \mathcal{C}^{1^{*}}\right)=\max \left\{p \cdot v \mid v \in \mathcal{C}^{1} \cap \mathbb{B}\right\} \quad \text { for any } p,
$$

and a similar formula is true for $d\left(p, \mathcal{C}^{2 *}\right)$. Now, bearing in mind (7), assume

$$
\delta^{H}\left(\mathcal{C}^{1^{*}}, \mathcal{C}^{2^{*}}\right)=d\left(p_{2}, \mathcal{C}^{1^{*}}\right)=p_{2} \cdot v_{2} \quad \text { for some } p_{2} \in \mathcal{C}^{2^{*}} \cap \mathbb{B}, v_{2} \in \mathcal{C}^{2} \cap \mathbb{B}
$$

then, exploiting again (4)

$$
\delta^{H}\left(\mathcal{C}^{1^{*}}, \mathcal{C}^{2^{*}}\right)=p_{2} \cdot v_{2} \leq d\left(v_{2}, \mathcal{C}^{1}\right)=d\left(v_{2}, \mathcal{C}^{1} \cap \mathbb{B}\right) \leq \delta^{H}\left(\mathcal{C}^{1}, \mathcal{C}^{2}\right)
$$

By interchanging the role of the cones appearing in the statement, we can complete the proof through the above argument.

We also obtain from Lemma 2.4, the Lipschitz character of the distance and signed distance functions from a cone:

Proposition 2.13. Let $\mathcal{C}^{1}, \mathcal{C}^{2} \in \operatorname{Cones}\left(\mathbb{R}^{N}\right), v \in \mathbb{R}^{N}$, then we have

$$
\begin{gathered}
\left|d\left(v, \mathcal{C}^{1}\right)-d\left(v, \mathcal{C}^{2}\right)\right| \leq|v| \delta^{H}\left(\mathcal{C}^{1}, \mathcal{C}^{2}\right) \\
\left|d^{\#}\left(v, \mathcal{C}^{1}\right)-d^{\#}\left(v, \mathcal{C}^{2}\right)\right| \leq 2|v| \delta^{H}\left(\mathcal{C}^{1}, \mathcal{C}^{2}\right) .
\end{gathered}
$$

It follows that both functions $(v, \mathcal{C}) \mapsto d(v, \mathcal{C})$ and $(v, \mathcal{C}) \mapsto d^{\#}(v, \mathcal{C})$ are Lipschitz on $R \mathbb{B} \times \operatorname{Cones}\left(\mathbb{R}^{N}\right)$ for any $R \geq 0$.

Proof. It suffices to show that

$$
\begin{aligned}
d\left(v, \mathcal{C}^{1}\right) & \leq d\left(v, \mathcal{C}^{2}\right)+|v| \delta^{H}\left(\mathcal{C}^{1}, \mathcal{C}^{2}\right) \\
d^{\#}\left(v, \mathcal{C}^{1}\right) & \leq d^{\#}\left(v, \mathcal{C}^{2}\right)+2|v| \delta^{H}\left(\mathcal{C}^{1}, \mathcal{C}^{2}\right) .
\end{aligned}
$$

By (4), we have

$$
d\left(v, \mathcal{C}^{1}\right)=p_{1} \cdot v \quad \text { for some } p_{1} \in \mathcal{C}^{1 *} \cap \mathbb{B} .
$$

We can find $p_{2} \in \mathcal{C}^{2 *} \cap \mathbb{B}$, with $\left|p_{1}-p_{2}\right|=d\left(p_{1}, \mathcal{C}^{2 *} \cap \mathbb{B}\right)$. Since $d\left(p_{1}, \mathcal{C}^{2 *} \cap \mathbb{B}\right) \leq$ $\delta^{H}\left(\mathcal{C}^{1 *}, \mathcal{C}^{2 *}\right)=\delta^{H}\left(\mathcal{C}^{1}, \mathcal{C}^{2}\right)$, we obtain

$$
\begin{aligned}
d\left(v, \mathcal{C}^{1}\right) & =p_{1} \cdot v \leq p_{2} \cdot v+\left|p_{2}-p_{1}\right||v| \\
& \leq d\left(v, \mathcal{C}^{2}\right)+|v| \delta^{H}\left(\mathcal{C}^{1}, \mathcal{C}^{2}\right) .
\end{aligned}
$$


This proves the part of the assertion above relative to the distances $d\left(\cdot, \mathcal{C}^{i}\right)$, $i=1,2$. By (5), we have

$$
d^{\#}\left(v, \mathcal{C}^{i}\right)=\max \left\{p \cdot v \mid p \in \mathcal{C}^{i} \cap \partial \mathbb{B}\right\} \quad i=1,2
$$

We adapt the same argument as above, and use Proposition 2.4 and Proposition 2.12. We have

$$
d^{\#}\left(v, \mathcal{C}^{1}\right)=\tilde{p}_{1} \cdot v, \text { for some } \tilde{p}_{1} \in \mathcal{C}^{1^{*}} \cap \partial \mathbb{B} .
$$

We denote by $\tilde{p}_{2}$ an element of $\mathcal{C}^{2^{*}} \cap \partial \mathbb{B}$ with $\left|\tilde{p}_{1}-\tilde{p}_{2}\right|=d\left(\tilde{p}_{1}, \mathcal{C}^{2^{*}} \cap \partial \mathbb{B}\right)$. We get

$$
\begin{aligned}
d^{\#}\left(v, \mathcal{C}^{1}\right) & =\tilde{p}_{1} \cdot v \leq \tilde{p}_{2} \cdot v+\left|\tilde{p}_{2}-\tilde{p}_{1}\right||v| \\
& \leq d^{\#}\left(v, \mathcal{C}^{2}\right)+|v| d\left(\tilde{p}_{1}, \mathcal{C}^{2^{*}} \cap \partial \mathbb{B}\right) .
\end{aligned}
$$

and

$$
\begin{aligned}
d^{\#}\left(v, \mathcal{C}^{1}\right) & \leq d^{\#}\left(v, \mathcal{C}^{2}\right)+|v| \max _{\tilde{p}_{1} \in \mathcal{C}^{1^{*}} \cap \partial \mathbb{B}} d\left(\tilde{p}_{1}, \mathcal{C}^{2^{*}} \cap \partial \mathbb{B}\right) \\
& \leq d^{\#}\left(v, \mathcal{C}^{2}\right)+2|v| \max _{\tilde{p}_{1} \in \mathcal{C}^{1^{*}} \cap \partial \mathbb{B}} d\left(\tilde{p}_{1}, \mathcal{C}^{2^{*}} \cap \mathbb{B}\right) \\
& \leq d^{\#}\left(v, \mathcal{C}^{2}\right)+2|v| \delta^{H}\left(\mathcal{C}^{1}, \mathcal{C}^{2}\right) .
\end{aligned}
$$

This proves the part of the assertion above relative the distances $d^{\#}\left(\cdot, \mathcal{C}^{i}\right) . i=$ 1,2 .

Since any distance function to a set have Lipschitz constant equal to 1 , we obtain for all $v_{1}, v_{2}$ in $R \mathbb{B}$

$$
\begin{aligned}
\left|d\left(v_{1}, \mathcal{C}^{1}\right)-d\left(v_{2}, \mathcal{C}^{2}\right)\right| & \leq\left|d\left(v_{1}, \mathcal{C}^{1}\right)-d\left(v_{2}, \mathcal{C}^{1}\right)\right|+\left|d\left(v_{2}, \mathcal{C}^{1}\right)-d\left(v_{2}, \mathcal{C}^{2}\right)\right| \\
& \leq\left|v_{1}-v_{2}\right|+R \delta^{H}\left(\mathcal{C}^{1}, \mathcal{C}^{2}\right) .
\end{aligned}
$$

In the same way, since the signed distance function have Lipschitz constant equal to 1 , we obtain for all $v_{1}, v_{2}$ in $R \mathbb{B}$

$$
\left|d^{\#}\left(v_{1}, \mathcal{C}^{1}\right)-d^{\#}\left(v_{2}, \mathcal{C}^{2}\right)\right| \leq\left|v_{1}-v_{2}\right|+2 R \delta^{H}\left(\mathcal{C}^{1}, \mathcal{C}^{2}\right)
$$

We deduce:

Corollary 2.14. The map $\mathcal{C} \mapsto \partial \mathcal{C} \cap \partial \mathbb{B}$ from $\operatorname{Cones}\left(\mathbb{R}^{N}\right)$ to the space of compact subsets of $\mathbb{R}^{N}$ endowed with the Hausdorff metric is Lipschitz with Lipschitz constant $\leq 2$.

Proof. Suppose $C$ is a closed subset of $\mathbb{R}^{N}$ and $v \in \mathbb{R}^{N}$. By Proposition 2.1 we have $\left|d^{\#}(v, C)\right|=d(v, \partial C)$. In particular $v \in \partial C$ if and only if $d^{\#}(v, C)=0$. 
Consider now a pair of cones $\mathcal{C}^{1}, \mathcal{C}^{2}$, if $v \in \partial \mathcal{C}^{1} \cap \partial \mathbb{B}$, then using Proposition 2.3 (vi) and what we said above, we obtain

$$
\begin{aligned}
d\left(v, \partial \mathcal{C}^{2} \cap \partial \mathbb{B}\right) & \leq 2 d\left(v, \partial \mathcal{C}^{2}\right) \\
& =2\left|d^{\#}\left(v, \mathcal{C}^{2}\right)\right| \\
& =2\left|d^{\#}\left(v, \mathcal{C}^{1}\right)-d^{\#}\left(v, \mathcal{C}^{2}\right)\right| \\
& \leq 2 \delta^{H}\left(\mathcal{C}^{1}, \mathcal{C}^{2}\right)
\end{aligned}
$$

where the last inequality used Proposition 2.13. Therefore

$$
\sup _{v \in \partial \mathcal{C}^{1} \cap \partial \mathbb{B}} d\left(v, \partial \mathcal{C}^{2} \cap \partial \mathbb{B}\right) \leq 2 \delta^{H}\left(\mathcal{C}^{1}, \mathcal{C}^{2}\right) .
$$

By symmetry, we obtain $d^{H}\left(\partial \mathcal{C}^{1} \cap \partial \mathbb{B}, \partial \mathcal{C}^{2} \cap \partial \mathbb{B}\right) \leq 2 \delta^{H}\left(\mathcal{C}^{1}, \mathcal{C}^{2}\right)$.

We proceed proving a compatibility result between the order relation $\succ$ and the metric $\delta^{H}$ in $\operatorname{Cones}\left(\mathbb{R}^{N}\right)$.

Theorem 2.15. Given $\mathcal{C} \in \operatorname{Cones}\left(\mathbb{R}^{N}\right)$ and $\varepsilon>0$, there are cones $\mathcal{C}^{\prime}, \mathcal{C}^{\prime \prime}$ satisfying $\delta^{H}\left(\mathcal{C}, \mathcal{C}^{\prime}\right)<\varepsilon, \delta^{H}\left(\mathcal{C}, \mathcal{C}^{\prime \prime}\right)<\varepsilon$ and $\mathcal{C}^{\prime} \prec \mathcal{C} \prec \mathcal{C}^{\prime \prime}$.

We first give some preliminary notions and results.

Given $\mathcal{C} \in \operatorname{Cones}\left(\mathbb{R}^{N}\right)$, we define the inradius $I(\mathcal{C})$ by

$$
I(\mathcal{C})=\max \{d(v, \partial \mathcal{C}) \mid v \in \mathcal{C} \cap \partial \mathbb{B}\}>0 .
$$

Lemma 2.16. The function $\left.I: \operatorname{Cones}\left(\mathbb{R}^{N}\right) \rightarrow\right] 0,+\infty[$ defined above is Lipschitz. Proof. On $\mathbb{B} \times \operatorname{Cones}\left(\mathbb{R}^{N}\right)$, the function $(v, \mathcal{C}) \mapsto d^{\#}(v, \mathcal{C})=d^{\#}(v, \mathbb{B} \cap \mathcal{C})$ is Lipschitz by by Proposition 2.13. Therefore by Lemma 2.9, the map $\mathcal{K}(\mathbb{B}) \times$ $\operatorname{Cones}\left(\mathbb{R}^{N}\right) \rightarrow \mathbb{R},(K, \mathcal{C}) \mapsto \min _{v \in K} d^{\#}(v, \mathbb{B} \cap \mathcal{C})$ is Lipschitz. To finish the proof of the lemma it suffices to observe that the map $\operatorname{Cones}\left(\mathbb{R}^{N}\right) \rightarrow \mathcal{K}(\mathbb{B}), \mathcal{C} \mapsto \mathbb{B} \cap \mathcal{C}$ is Lipschitz by Lemma 2.11 .

For $\delta \geq 0$, we set

$$
\mathcal{C}^{-\delta}=\left\{v\left|d^{\#}(v, \mathcal{C})+\delta\right| v \mid \leq 0\right\}
$$

Lemma 2.17. Given $\mathcal{C} \in \operatorname{Cones}\left(\mathbb{R}^{N}\right)$. We have:

(i) $\mathcal{C}^{-0}=\mathcal{C}$.

(ii) The subset $\mathcal{C}^{-\delta}$ of $\mathbb{R}^{N}$ is a cone for any $\delta \in[0, I(\mathcal{C})[$.

(ii) For $\delta_{2}<\delta_{1}$, we have $\mathcal{C}^{-\delta_{1}} \prec \mathcal{C}^{-\delta_{2}}$.

Moreover, the map $(\delta, \mathcal{C}) \rightarrow \mathcal{C}^{-\delta} \in \operatorname{Cones}\left(\mathbb{R}^{N}\right)$ is locally Lipschitz on the open subset $\{(\delta, \mathcal{C}) \mid 0 \leq \delta<I(\mathcal{C})\} \subset\left[0,+\infty\left[\times \operatorname{Cones}\left(\mathbb{R}^{N}\right)\right.\right.$. 
Proof. The fact that $\mathcal{C}^{-0}=\mathcal{C}$ is obvious. The set $\mathcal{C}^{-\delta}$, being contained in $\mathcal{C}$, does not contain any affine complete line, moreover its interior is non-empty, since any $v \in \mathcal{C} \cap \partial \mathbb{B}$ whose signed distance from $\mathcal{C}$ realizes $I(\mathcal{C})$, up to a sign, belongs to it. Finally, $\mathcal{C}^{-\delta}$ is a closed convex cone with vertex at 0 since it is the 0 -sublevel of a continuous convex positively homogeneous function. The relation $\mathcal{C}^{-\delta_{2}} \prec \mathcal{C}^{-\delta_{1}}$, for $\delta_{2}<\delta_{1}$, is immediate, since for $v \in \mathcal{C}^{-\delta_{2}} \backslash\{0\}$, we have

$$
d^{\#}(v, \mathcal{C})+\delta_{1}|v|=d^{\#}(v, \mathcal{C})+\delta_{2}|v|+\left(d_{2}-d_{1}\right)|v| \leq\left(d_{2}-d_{1}\right)|v|<0 .
$$

It remains to show that the map $(\delta, \mathcal{C}) \rightarrow \mathcal{C}^{-\delta}$ is locally Lipschitz on the open subset $\{(\delta, \mathcal{C}) \mid 0 \leq \delta<I(C)\}$ of $\left[0,+\infty\left[\times \operatorname{Cones}\left(\mathbb{R}^{N}\right)\right.\right.$. For this we remark that the map $f: \mathbb{B} \times\left(\left[0,+\infty\left[\times\right.\right.\right.$ Cones $\left.\left(\mathbb{R}^{N}\right)\right) \rightarrow \mathbb{R}, v \mapsto d^{\#}(v, \mathcal{C})+\delta|v|$ is Lipschitz, and convex in $v$. Since we have $\inf _{v \in B} f(v,(\delta, \mathcal{C}))<0$, for $0 \leq \delta<I(C)$, it follows from Lemma 2.10 that $\mathcal{C}^{-\delta} \cap \mathbb{B}=\{v \in \mathbb{B} \mid f(v,(\delta, \mathcal{C})) \leq \delta\}$ is a locally Lipschitz function of $(\delta, \mathcal{C})$ on the set $0 \leq \delta<I(C)$.

Proof of Theorem 2.15. It is enough to show that there is $\mathcal{C}^{\prime}$ with $\delta^{H}\left(\mathcal{C}, \mathcal{C}^{\prime}\right)<\varepsilon$ and $\mathcal{C}^{\prime} \prec \mathcal{C}$. The existence of $\mathcal{C}^{\prime \prime}$ with the properties asserted in the statement then comes by duality taking into account Proposition 2.6 and the fact that $\mathcal{C}^{0} \mapsto \mathcal{C}^{0^{*}}$ is an isometry by Proposition 2.12.

The existence of $\mathcal{C}^{\prime}$ now follows from Lemma 2.17, since for $\left.\delta \in\right] 0, I(C)$ [, we have $\mathcal{C}^{-\delta} \prec \mathcal{C}$, and $\mathcal{C}^{-\delta} \rightarrow \mathcal{C}$, as $\delta \rightarrow 0$.

For $\mathcal{C}, \mathcal{C}^{\prime} \in \operatorname{Cones}\left(\mathbb{R}^{N}\right)$, we define

$$
D^{\#}\left(\mathcal{C}, \mathcal{C}^{\prime}\right)=\sup _{x \in \mathcal{C} \cap \partial \mathbb{B}} d^{\#}\left(x, \mathcal{C}^{\prime}\right) .
$$

Note that $D^{\#}\left(\mathcal{C}, \mathcal{C}^{\prime}\right) \leq 0$ if and only if $\mathcal{C} \subset \mathcal{C}^{\prime}$, and $D^{\#}\left(\mathcal{C}, \mathcal{C}^{\prime}\right)<0$ if and only if $\mathcal{C} \prec \mathcal{C}^{\prime}$.

Lemma 2.18. The function $D^{\#}: \operatorname{Cones}\left(\mathbb{R}^{N}\right) \times \operatorname{Cones}\left(\mathbb{R}^{N}\right),\left(\mathcal{C}, \mathcal{C}^{\prime}\right) \mapsto D^{\#}\left(\mathcal{C}, \mathcal{C}^{\prime}\right)$ is Lipschitz. In particular, the subset $\left\{\left(\mathcal{C}, \mathcal{C}^{\prime}\right) \mid \mathcal{C} \prec \mathcal{C}^{\prime}\right\}$ is open in $\operatorname{Cones}\left(\mathbb{R}^{N}\right) \times$ $\operatorname{Cones}\left(\mathbb{R}^{N}\right)$

Proof. It is very similar to the proof of Lemma 2.16. In the proof of that Lemma we obtained that the $\operatorname{map} \mathcal{K}(\mathbb{B}) \times \operatorname{Cones}\left(\mathbb{R}^{N}\right) \rightarrow \mathbb{R},\left(K, \mathcal{C}^{\prime}\right) \mapsto \min _{v \in K} d^{\#}\left(v, \mathbb{B} \cap \mathcal{C}^{\prime}\right)$ is Lipschitz. Again to finish the proof of the first part of the lemma it suffices to observe that the map $\operatorname{Cones}\left(\mathbb{R}^{N}\right) \rightarrow \mathcal{K}(\mathbb{B}), \mathcal{C} \mapsto \mathbb{B} \cap \mathcal{C}$ is Lipschitz by Lemma 2.11 .

The last part of the lemma is now clear since $\left\{\left(\mathcal{C}, \mathcal{C}^{\prime}\right) \mid \mathcal{C} \prec \mathcal{C}^{\prime}\right\}=\left\{\left(\mathcal{C}, \mathcal{C}^{\prime}\right) \mid\right.$ $\left.D^{\#}\left(\mathcal{C}, \mathcal{C}^{\prime}\right)<0\right\}$

We now get the continuity of the action of the linear group on cones. We use, as usual, the symbol GL( $\left.\mathbb{R}^{N}\right)$ to denote the group of linear invertible maps from 
$\mathbb{R}^{N}$ to itself. We endow it with the operator norm, denoted, like the Euclidean norm in $\mathbb{R}^{N}$, by $|\cdot|$ and defined, for any $T \in \mathrm{GL}\left(\mathbb{R}^{N}\right)$ as

$$
|T|=\max \{|T x| \mid x \in \mathbb{B}\} .
$$

It is clear that $T \mathcal{C} \in \operatorname{Cones}\left(\mathbb{R}^{N}\right)$ for any $\mathcal{C} \in \operatorname{Cones}\left(\mathbb{R}^{N}\right)$, and any $T \in \operatorname{GL}\left(\mathbb{R}^{N}\right)$.

Lemma 2.19. The map $\mathrm{GL}\left(\mathbb{R}^{N}\right) \times \operatorname{Cones}\left(\mathbb{R}^{N}\right) \rightarrow \operatorname{Cones}\left(\mathbb{R}^{N}\right),(T, \mathcal{C}) \mapsto T \mathcal{C}$, is locally Lipschitz. More precisely, if we set for $K \geq 1$

$$
G_{K}=\left\{T \in \mathrm{GL}\left(\mathbb{R}^{N}\right)|| T \mid \leq K, \text { and }\left|T^{-1}\right| \leq K\right\}
$$

then we have for all $T, T^{\prime} \in G_{K}, \mathcal{C}, \mathcal{C}^{\prime} \in \operatorname{Cones}\left(\mathbb{R}^{N}\right)$

$$
\delta^{H}\left(T \mathcal{C}, T^{\prime} \mathcal{C}^{\prime}\right) \leq K\left|T-T^{\prime}\right|+K^{2} \delta^{H}\left(\mathcal{C}, \mathcal{C}^{\prime}\right)
$$

Proof. We consider $T, T^{\prime} \in G_{K}, \mathcal{C}, \mathcal{C}^{\prime} \in \operatorname{Cones}\left(\mathbb{R}^{N}\right)$, and fix $x \in \mathbb{B}$. The idea is to exploit the formula (8), therefore, since $x$ has been arbitrarily taken in $\mathbb{B}$, instead of (12) we will equivalently show

$$
\left|d(x, T \mathcal{C} \cap \mathbb{B})-d\left(x, T^{\prime} \mathcal{C}^{\prime} \cap \mathbb{B}\right)\right| \leq K\left|T-T^{\prime}\right|+K^{2} \delta^{H}\left(\mathcal{C}, \mathcal{C}^{\prime}\right) .
$$

There exists $y^{\prime} \in \mathcal{C}^{\prime}$ with $\left|T^{\prime} y^{\prime}\right| \leq 1$ such that $d\left(x, T^{\prime} \mathcal{C}^{\prime} \cap \mathbb{B}\right)=\left|x-T^{\prime} y^{\prime}\right|$. The fact that $T^{\prime} \in G_{K}$ implies that $\left|y^{\prime}\right| \leq K$. We have

$$
\left|x-T y^{\prime}\right| \leq\left|x-T^{\prime} y^{\prime}\right|+\left|T^{\prime}-T\right|\left|y^{\prime}\right| \leq d\left(x, T^{\prime} \mathcal{C}^{\prime} \cap \mathbb{B}\right)+K\left|T^{\prime}-T\right| .
$$

Since $y^{\prime} \in \mathcal{C}^{\prime}$ and $\left|y^{\prime}\right| \leq K$, we can find $y \in \mathcal{C}$ such that $|y| \leq K$ and $\left|y-y^{\prime}\right| \leq$ $K \delta^{H}\left(\mathcal{C}, \mathcal{C}^{\prime}\right)$. Therefore we obtain

$$
\begin{aligned}
|x-T y| & \leq\left|x-T y^{\prime}\right|+\left|T y^{\prime}-T y\right| \\
& \leq d\left(x, T^{\prime} \mathcal{C}^{\prime} \cap \mathbb{B}\right)+K\left|T^{\prime}-T\right|+|T|\left|y-y^{\prime}\right| \\
& \leq d\left(x, T^{\prime} \mathcal{C}^{\prime} \cap \mathbb{B}\right)+K\left|T^{\prime}-T\right|+K^{2} \delta^{H}\left(\mathcal{C}, \mathcal{C}^{\prime}\right) .
\end{aligned}
$$

Since $|x| \leq 1$, by Proposition 2.3, we have

$$
d(x, T \mathcal{C} \cap \mathbb{B})=d(x, T C) \leq|x-T y|,
$$

and consequently

$$
d(x, T \mathcal{C} \cap \mathbb{B})-d\left(x, T^{\prime} \mathcal{C}^{\prime} \cap \mathbb{B}\right) \leq K\left|T^{\prime}-T\right|+K^{2} \delta^{H}\left(\mathcal{C}, \mathcal{C}^{\prime}\right) .
$$

By exchanging the role of $\mathcal{C}$ an $\mathcal{C}^{\prime}$, we in the end obtain (13). 


\subsection{Cone Structures on open subsets of $\mathbb{R}^{N}$}

Definition 2.20. A cone structure (resp. Lipschitz cone structure) on an open subset $U$ of $\mathbb{R}^{N}$ is a family of cones $\mathcal{C}=\left(\mathcal{C}_{x}\right)_{x \in U}$ with $\mathcal{C}_{x} \in \operatorname{Cones}\left(\mathbb{R}^{N}\right)$, and the map $U \rightarrow$ Cones $\left(\mathbb{R}^{N}\right), x \mapsto \mathcal{C}_{x}$ continuous (resp. locally Lipschitz), where, as we said above, Cones $\left(\mathbb{R}^{N}\right)$ is endowed with the distance $\delta^{H}$ and the topology inherited from that distance.

If $\varphi: U \rightarrow V$ is a $\mathrm{C}^{1}$ diffeomorphism between two open subsets of $\mathbb{R}^{N}$, and $\mathcal{C}=\left(\mathcal{C}_{x}\right)_{x \in U}$ is a cone structure on $U$, we define the family of cones $\varphi_{*} \mathcal{C}$ on $V$ by

$$
\varphi_{*} \mathcal{C}_{y}=D_{x} \varphi\left(\mathcal{C}_{x}\right), \text { with } \varphi(x)=y \text {. }
$$

The following proposition is now clearly a consequence of Lemma 2.19

Lemma 2.21. The family of cones $\varphi_{*} \mathcal{C}$ is a (continuous) cone structure on $V$. Moreover, if $\mathcal{C}=\left(\mathcal{C}_{x}\right)_{x \in U}$ is a Lipschitz cone structure, so is $\varphi_{*} \mathcal{C}$.

By Lemma 2.12, if $\mathcal{C}$ is a cone structure on $U \subset \mathbb{R}^{N}$, the dual family $\mathcal{C}^{*}$ defined by $\mathcal{C}_{x}^{*}=\left(\mathcal{C}_{x}\right)_{x \in U}^{*}$ is also a cone structure on $U$. Note that for a Lipschitz cone structure $\mathcal{C}$ the dual $\mathcal{C}^{*}$ is also Lipschitz by Proposition 2.12 .

We recall the notions of $\mathcal{C}$-causal and $\mathcal{C}$-future directed curves given in the Introduction.

Definition 2.22. If $\mathcal{C}$ is a cone structure on the open subset $U$ of $\mathbb{R}^{N}$, a Lipschitz curve $\xi:[a, b] \rightarrow U$ is $\mathcal{C}$-causal (resp. $\mathcal{C}$-future directed) if $\dot{\xi}(t) \in \mathcal{C}_{\xi(t)}$ (resp. $\left.\dot{\xi}(t) \in \dot{\mathcal{C}}_{\xi(t)}\right)$ for almost every $t \in[a, b]$.

We recall the a Lipschitz curve has a derivative almost everywhere, so the notions above make sense.

We now define the function $d_{\mathcal{C}}: U \times \mathbb{R}^{N} \rightarrow[0,+\infty[$ by

$$
d_{\mathcal{C}}(x, v)=d\left(v, \mathcal{C}_{x}\right)
$$

Since the function $d_{\mathcal{C}}$ is non negative and positively homogeneous, we can use it to define an intrinsic length of a curve. Namely, given a curve $\xi:[a, b] \rightarrow \mathbb{R}^{N}$, we set

$$
\int_{\xi} d_{\mathcal{C}}=\int_{a}^{b} d_{\mathcal{C}}(\xi(t), \dot{\xi}(t)) d t=\int_{a}^{b} d\left(\dot{\xi}(t), \mathcal{C}_{\xi(t)}\right) d t .
$$

Notice that the above integral is invariant for orientation-preserving change of parameter, as an intrinsic length should be. Obviously a curve $\xi$ is $\mathcal{C}$-causal if and only if $\int_{\xi} d_{\mathcal{C}}=0$.

We extend the relations $\prec$ to cone structures in the obvious way. Namely if $\mathcal{C}$ and $\mathcal{C}^{\prime}$ are cone structures on $U$ we say that $\mathcal{C} \prec \mathcal{C}^{\prime}$ if we have $\mathcal{C}_{x} \prec \mathcal{C}_{x}^{\prime}$, for every $x \in U$. 
Lemma 2.23. Let $\mathcal{C}, \mathcal{C}^{\prime}$ be two cone structures on the open subset $U \subset \mathbb{R}^{N}$ with $\mathcal{C} \prec \mathcal{C}^{\prime}$. Given $x_{0} \in U$, we can find a neighborhood $V$ of $x_{0}$ in $U$ and $\mathcal{C}_{1}, \mathcal{C}_{2} \in \operatorname{Cones}\left(\mathbb{R}^{N}\right)$ such that

$$
\mathcal{C}_{x} \prec \mathcal{C}_{1} \prec \mathcal{C}_{2} \prec \mathcal{C}_{x}^{\prime} \text {, for every } x \in V \text {. }
$$

Proof. We will first find $\mathcal{C}_{1}$ and $\mathcal{C}_{2}$ such that

$$
\mathcal{C}_{x_{0}} \prec \mathcal{C}_{1} \prec \mathcal{C}_{2} \prec \mathcal{C}_{x_{0}}^{\prime} .
$$

According Lemma 2.17 we have $\left(\mathcal{C}_{x_{0}}^{\prime}\right)^{-\delta} \prec \mathcal{C}_{x_{0}}$, for $\delta>0$, and $\left(\mathcal{C}_{x_{0}}^{\prime}\right)^{-\delta} \rightarrow \mathcal{C}_{x_{0}}^{\prime}$, for $\delta \rightarrow 0$. It therefore follows from Lemma 2.18 that $\left(\mathcal{C}_{x_{0}}^{\prime}\right)^{-\delta} \succ \mathcal{C}_{x_{0}}$, for $\delta$ small enough. Therefore we can take $\mathcal{C}_{2}=\left(\mathcal{C}_{x_{0}}^{\prime}\right)^{-\delta}$ for some appropriate $\delta>0$. To obtain $\mathcal{C}_{1}$, it suffices to apply what we just did with $C_{x_{0}}, \mathcal{C}_{2}$ instead of $C_{x_{0}}, C_{x_{0}}^{\prime}$.

By the last part of Lemma 2.18 and the continuity of $x \mapsto \mathcal{C}_{x}, C_{x}^{\prime}$, both sets $V^{\prime}=\left\{\mathcal{C}_{x} \mid \mathcal{C}_{x} \prec C_{1}\right\}$ and $V^{\prime \prime}=\left\{\mathcal{C}_{x}^{\prime} \mid \mathcal{C}_{x}^{\prime} \succ C_{2}\right\}$ are open. Since they contain $x_{0}$, we can then take $V=V^{\prime} \cap V^{\prime \prime}$.

The next result is about curves with intrinsic length small with respect to the Euclidean length. It will be of crucial importance for the proof of Theorem 1.1.

Proposition 2.24. Let $\mathcal{C}_{x}, \mathcal{C}_{x}^{\prime}$ be continuous cone structures defined on a neighborhood of a compact convex subset $K$ of $\mathbb{R}^{N}$ with $\mathcal{C} \prec \mathcal{C}^{\prime}$. There exist positive constants $\delta, \rho$ such that for any nonconstant curve $\gamma:[a, b] \rightarrow K$, with

$$
\int_{\gamma} d_{\mathcal{C}}<\min \{\rho, \delta \ell(\gamma)\},
$$

we can find a $\mathcal{C}^{\prime}$-future directed piecewise linear curve lying in $K$ which joins $\gamma(a)$ to $\gamma(b)$.

Proof. By Lemma 2.23 we can construct a finite covering of $K$ by open Euclidean balls $x_{i}+3 \eta \mathbb{B}, i=1, \ldots, k$, for some $\eta>0, x_{i} \in K$, such that for any $i$ we can find cones $\mathcal{C}_{1}^{i}, \mathcal{C}_{2}^{i}$ satisfying

$$
\mathcal{C}_{y}^{\prime} \succ \mathcal{C}_{2}^{i} \succ \mathcal{C}_{1}^{i} \succ \mathcal{C}_{y} \quad \text { for any } y \in x_{i}+3 \eta \mathbb{B} .
$$

We denote by $\delta_{i}$ a constant satisfying (6) with $\mathcal{C}_{1}^{i}, \mathcal{C}_{2}^{i}$ in place of $\mathcal{C}^{1}, \mathcal{C}^{2}$, respectively, $i=1, \cdots, k$, and set $\delta=\min _{i} \delta_{i}, \rho=\eta \delta$. Note that the assertion is true for any curve $\xi:[c, d] \rightarrow x_{i}+3 \eta \mathbb{B}$, for some $i$, satisfying

$$
\int_{\xi} d_{\mathcal{C}}<\delta \ell(\xi),
$$

because in this case we infer from (17), Lemma 2.7, and the convex character of $K$, that the oriented segment from $\xi(c)$ to $\xi(d)$ is a $\mathcal{C}^{\prime}$-future directed curve. 
Given $\gamma:[a, b] \rightarrow K$, the idea is to define inductively an increasing finite sequence of times

$$
t_{0}=a, t_{1}, \cdots, t_{m-1}, t_{m}=b
$$

such that $\left.\gamma\right|_{\left[t_{j-1}, t_{j}\right]} \subset x_{i_{j}}+3 \eta \mathbb{B}$, for some index $i_{j}$, and then to argue on each subinterval.

We assume that $\gamma(a) \in x_{i_{1}}+\eta \stackrel{\circ}{\mathbb{B}}$ and denote by $t_{1}$ the largest $t \in[a, b]$ such that $\gamma([a, t]) \subset \gamma(a)+\eta \mathbb{B}$. If $\left|\gamma(a)-\gamma\left(t_{1}\right)\right|<\eta$ then $t_{1}=b$, and the support of the curve $\gamma$ is entirely contained in $x_{i_{1}}+2 \eta \mathbb{B}$, so that the assertion is obtained according to what we pointed out above. If instead $\left|\gamma(a)-\gamma\left(t_{1}\right)\right|=\eta$ then by the assumption

$$
\int_{\left.\gamma\right|_{\left[a, t_{1}\right]}} d_{\mathcal{C}} \leq \int_{\gamma} d_{\mathcal{C}} \leq \rho=\eta \delta \leq \delta \ell\left(\left.\gamma\right|_{\left[a, t_{1}\right]}\right),
$$

which implies that the oriented segment from $\gamma(a)$ to $\gamma\left(t_{1}\right)$ is $\mathcal{C}^{\prime}$-future directed, since $\gamma\left(\left[a, t_{1}\right]\right) \subset x_{i_{1}}+2 \eta \mathbb{B}$.

If $t_{1}<b$, using the same process as above, we construct by induction a sequence $t_{0}=a, t_{1}, \ldots, t_{k}$ such that $\left|\gamma\left(t_{j}\right)-\gamma\left(t_{j+1}\right)\right|=\eta$, and the oriented segment from $\gamma\left(t_{j}\right)$ to $\gamma\left(t_{j+1}\right)$ is $\mathcal{C}^{\prime}$-future directed continuous $\mathrm{C}^{\infty}$ curve from $\gamma\left(t_{j}\right)$ to $\gamma\left(t_{j+1}\right)$, for $j=0, \ldots, k-1$. Since $\gamma$ has finite length, there must be $m>1$ with $t_{m}=b$. Some precautions must be taken for the last step to complete the proof. In fact we cannot estimate the length of $\gamma \mid\left[t_{m-1}, t_{m}\right]$, and so we argue on $\gamma \mid\left[t_{m-2}, t_{m}\right]$. Note that $\gamma\left(\left[t_{m-2}, t_{m}\right]\right) \subset x_{i_{m-2}}+3 \eta \mathbb{B}$, for some $i_{m-2}$, and $\ell\left(\left.\gamma\right|_{\left[t_{m-2}, t_{m}\right]}\right) \geq \eta$, and we can repeat the estimate $(18)$, with $\left[t_{m-2}, t_{m}\right]$ in place of $\left[0, t_{1}\right]$, to conclude the argument.

\subsection{Cone Structures on a Manifold}

Let $M$ be a connected, paracompact, $\mathrm{C}^{\infty}$ manifold of dimension $N$. We denote, for any $x \in M$, by $T_{x} M, T_{x}^{*} M$ the tangent and cotangent spaces of $M$ at $x$, respectively. The tangent and cotangent bundle are $T M$ and $T^{*} M$, respectively. We will assume in the sequel that $M$ is endowed with a Riemannian metric $g$.

We proceed introducing some notations.

For $v, v^{\prime} \in T_{x} M$, we will set $|v|_{x}=\left(g_{x}(v, v)\right)^{1 / 2}$. We will also denote by $|\cdot|_{x}$ the norm on $T_{x}^{*} M$ dual to $|\cdot|_{x}$ on $T_{x} M$. The formula $d_{x}^{g}\left(v, v^{\prime}\right)=\left|v-v^{\prime}\right|_{x}$ gives a distance on $T_{x} M$, we will denote the corresponding closed unit ball by $\mathbb{B}_{x}$. We will write $d_{g}(x, y)$ for the Riemannian distance between the points $x, y \in M$, and denote by $B_{g}(x, r)$ the related metric closed ball centered at $x$, with radius $r>0$.

Definition 2.25. A (resp. Lipschitz) cone structure $\mathcal{C}$ on $M$ is a map which associates to any $x \in M$ a (complete) cone $\mathcal{C}_{x} \subset T_{x} M$, such that for any $\mathrm{C}^{\infty}$ chart $\varphi: U \rightarrow \varphi(U) \subset \mathbb{R}^{N}$ the family of cones $\varphi_{*} \mathcal{C}$ defined by

$$
\left(\varphi_{*} \mathcal{C}\right)_{y}=D \varphi\left(\varphi^{-1}(y)\right) \mathcal{C}_{\varphi^{-1}(y)}
$$


is a continuous (resp. locally Lipschitz) family of cones on the open subset $\varphi(U) \subset$ $\mathbb{R}^{N}$.

In the definition above $D \varphi(x): T_{x} M \rightarrow T_{\varphi(x)} \mathbb{R}^{N}=\mathbb{R}^{N}$, is the derivative (or tangent map) of $\varphi$ at $x \in U$.

By Lemma 2.21, as usual, to verify that the family of cone $\mathcal{C}$ is a (resp. Lipschitz) cone structure, it is sufficient to find family of charts $\left(U_{i}, \varphi_{i}\right)_{i \in I}$ such that $\varphi_{i *} \mathcal{C}$ is a (resp. Lipschitz) cone structure on the open subset $\varphi_{i}\left(U_{i}\right)$ of $\mathbb{R}^{N}$, for each $i \in I$.

In the same way we can define the notion of (resp. Lipschitz) dual cone structure on $M$. A dual cone structure $\tilde{\mathcal{C}}$ is a family $\left(\tilde{\mathcal{C}}_{x}\right)_{x \in M}$, where $\tilde{\mathcal{C}}_{x} \subset T_{x}^{*} M$ is a (complete) cone for each $x \in M$, such that or any $\mathrm{C}^{\infty}$ chart $\varphi: U \rightarrow \varphi(U) \subset \mathbb{R}^{N}$ the family of cones $\varphi^{*} \tilde{\mathcal{C}}$ defined by

$$
\left(\varphi^{*} \tilde{\mathcal{C}}\right)_{y}=\left[{ }^{t} D \varphi\left((\varphi)^{-1}(y)\right)\right]^{-1} \tilde{\mathcal{C}}_{(\varphi)^{-1}(y)} .
$$

is a continuous (resp. locally Lipschitz) family of cones on the open subset $\varphi(U) \subset$ $\mathbb{R}^{N}$. Here we denote by ${ }^{t} D \varphi(x):\left(\mathbb{R}^{N}\right)^{*} \rightarrow T_{x}^{*} M$, the transpose (or dual) of the the derivative $D \varphi(x): T_{x} M \rightarrow \mathbb{R}^{N}$, and we identified $\left(\mathbb{R}^{N}\right)^{*}$ to $\mathbb{R}^{N}$ using the canonical basis (or the canonical Euclidean product) as we did since the beginning of $\S 2.1$. Again by the dual analog of Lemma 2.21, as usual, to verify that the family of cone $\tilde{\mathcal{C}}$ is a (resp. Lipschitz) dual cone structure, it is sufficient to find family of charts $\left(U_{i}, \varphi_{i}\right)_{i \in I}$ such that $\varphi_{i}{ }^{*} \tilde{\mathcal{C}}$ is a (resp. Lipschitz) cone structure on the open subset $\varphi_{i}\left(U_{i}\right)$ of $\mathbb{R}^{N}$, for each $i \in I$.

We now extend to cone structures on $M$ the notion of dual cone. Given $x \in M$ and a cone $\mathcal{C}^{0} \subset T_{x} M$ we define the dual (or polar) $\mathcal{C}^{0^{*}} \subset T_{x}^{*} M$ via

$$
\mathcal{C}^{0^{*}}=\left\{p \in T_{x}^{*} M \mid p(v) \leq 0 \text { for } v \in \mathcal{C}^{0}\right\} .
$$

Therefore if $\mathcal{C}$ is a cone structure on $M$, the dual cone structure is $\mathcal{C}^{*}$ defined by $\mathcal{C}_{x}^{*}=\left(\mathcal{C}_{x}\right)^{*}$. It is not difficult to check, with the notations above, that for a chart $\varphi: U \rightarrow \varphi(U) \subset \mathbb{R}^{N}$, the cones $\varphi_{*} \mathcal{C}_{y}$ and $\varphi^{*} \mathcal{C}_{y}^{*}$ are dual cones in $\mathbb{R}^{N}$ for every $y \in \varphi(U)$. It follows that $\mathcal{C}^{*}$ is a (resp. Lipschitz) dual cone structure on $M$ if $\mathcal{C}$ is a (resp. Lipschitz) cone structure.

The order relation $\succ$ for cone structures is naturally extended in this setting. If $\mathcal{C}, \mathcal{C}^{\prime}$ are cone structures on $M$, we say say that $\mathcal{C} \prec \mathcal{C}^{\prime}$ if $\mathcal{C}_{x} \prec \mathcal{C}_{x}^{\prime}$, for every $x \in M$. Same thing for dual cone structures. Of course $\mathcal{C} \prec \mathcal{C}^{\prime}$ if and only if $\mathcal{C}^{*} \succ \mathcal{C}^{*}$ by

We now introduce the function $d_{\mathcal{C}}^{g}, d_{\mathcal{C}}^{g \#}: T M \rightarrow \mathbb{R}$ defined for all $(x, v) \in T M$

$$
\begin{aligned}
d_{\mathcal{C}}^{g}(x, v) & =d_{x}^{g}\left(v, \mathcal{C}_{x}\right)=\inf _{v^{\prime} \in \mathcal{C}_{x}} d_{x}^{g}\left(v, v^{\prime}\right) . \\
d_{\mathcal{C}}^{g \#}(x, v) & =2 d_{x}^{g}\left(v, \mathcal{C}_{x}\right)-d_{x}^{g}\left(v, \partial \mathcal{C}_{x}\right) .
\end{aligned}
$$


By Lemma 2.4 we have

$$
\begin{aligned}
d_{\mathcal{C}}^{g}(x, v) & =\sup \left\{\left.p(v)\left|p \in \mathcal{C}_{x}^{*},\right| p\right|_{x} \leq 1\right\} \\
d_{\mathcal{C}}^{g \#}(x, v) & =\sup \left\{\left.p(v)\left|p \in \mathcal{C}_{x}^{*},\right| p\right|_{x}=1\right\} .
\end{aligned}
$$

We can now introduce the function $I_{g}(\mathcal{C})=M \rightarrow[0,+\infty[$ defined by

$$
I_{g}(\mathcal{C})(x)=-\min \left\{d_{\mathcal{C}}^{g \#}(x, v) \mid v \in \mathcal{C}_{x}, \cap \mathbb{B}_{x}\right\}
$$

For $\delta \geq 0$, we also introduce the family $\mathcal{C}^{-\delta}$ defined by

$$
\mathcal{C}_{x}^{-\delta}=\left\{\left.v \in T_{x} M\left|d_{\mathcal{C}}^{g \#}(x, v) \leq-\delta\right| v\right|_{x}\right\}
$$

For each $\delta<I_{g}(C)(x)$, we know that $\mathcal{C}_{x}^{-\delta}$ is a cone in $T_{x} M$. If moreover $\delta>0$, we have $\mathcal{C}_{x}^{-\delta} \prec \mathcal{C}_{x}$.

If $x \in M$, and $\mathcal{C}_{1}, \mathcal{C}_{2}$ are two cones in $T_{x} M$, we define a Hausdorff-type distance between them, by

$$
\delta_{x}^{g, H}\left(\mathcal{C}_{1} \mathcal{C}_{2}\right)=\max \left\{\max _{w \in \mathcal{C}_{2} \cap \mathbb{B}_{x}} d_{x}^{g}\left(w, \mathcal{C}_{1}\right), \max _{v \in \mathcal{C}_{1} \cap \mathbb{B}_{x}} d_{x}^{g}\left(v, \mathcal{C}_{2}\right)\right\}
$$

Proposition 2.26. The map $T M \rightarrow\left[0,+\infty\left[,(x, v) \mapsto|v|_{x}\right.\right.$ is locally Lipschitz on $T M$.

Let $\mathcal{C}$ be a (resp. Lipschitz) cone structures on the Riemannian manifold $M$, the following functions are continuous (resp. locally Lipschitz):

(i) $(x, v) \mapsto d_{\mathcal{C}}^{g}(x, v)$, from $T M$ to $[0,+\infty[$;

(ii) $(x, v) \mapsto d_{\mathcal{C}}^{g \#}(x, v)$, from $T M$ to $\mathbb{R}$;

(iii) $I_{g}(\mathcal{C}): M \rightarrow[0,+\infty[$, in particular, the subset $\{(\delta, x) \in[0,+\infty[\times M \mid \delta<$ $\left.I_{g}(\mathcal{C})(x)\right\}$ is open in $[0,+\infty[\times M$;

(iv) $(\delta, x) \mapsto \delta_{x}^{g, H}\left(\mathcal{C}_{x}^{-\delta}, \mathcal{C}_{x}\right)$ from the open subset $\{(\delta, x) \in[0,+\infty[\times M \mid \delta<$ $\left.I_{g}(\mathcal{C})(x)\right\}$ to $[0,+\infty[;$

If $\theta: M \rightarrow\left[0,+\infty\left[\right.\right.$ is continuous (resp. locally Lipschitz), with $\theta(x)<I_{g}(\mathcal{C})(x)$, for every $x \in M$, then $\mathcal{C}^{-\theta}=\left(\mathcal{C}_{x}^{-\theta(x)}\right)_{x \in M}$ is a (resp. Lipschitz) cone structure on $M$.

If $\mathcal{C}^{\prime}$ is another cone structure on $M$, then the sets $\{(\delta, x) \in[0,+\infty[\times M \mid \delta<$ $\left.I_{g}(\mathcal{C})(x), \mathcal{C}_{x}^{\prime} \prec \mathcal{C}_{x}^{-\delta}\right\}$ and $\left\{(\delta, x) \in\left[0,+\infty\left[\times M \mid \delta<I_{g}(\mathcal{C})(x), \mathcal{C}_{x}^{\prime} \succ \mathcal{C}_{x}^{-\delta}\right\}\right.\right.$ are open in $[0,+\infty[\times M$. 
The easiest way to prove this proposition is to use the Gram-Schmidt orthogonalization method. This method provides a way to choose an orthonormal basis for each scalar product on $\mathbb{R}^{N}$, depending smoothly on the scalar product. Define $Q^{+}\left(\mathbb{R}^{N}\right)$ as the space of positive definite bilinear forms (or scalar products) on $\mathbb{R}^{N}$.

Proposition 2.27 (Gram-Schmidt). There exist $\mathrm{C}^{\infty}$ (even real analytic) maps $Q^{+}\left(\mathbb{R}^{N}\right) \rightarrow \mathbb{R}^{N}, g \mapsto e_{i}^{g}, i=1, \ldots, N$ such that $\left(e_{1}^{g}, \cdots, e_{N}^{g}\right)$ is a basis of $\mathbb{R}^{N}$ orthonormal for the scalar product $g$.

In particular if we define $L^{g}: \mathbb{R}^{N} \rightarrow \mathbb{R}^{N}$ by $L^{g}\left(x_{1}, \ldots, x_{N}\right)=\sum_{i=1}^{N} x_{i} e_{i}^{g}$, then $L^{g}$ is an isometry of $\mathbb{R}^{N}$ with the usual scalar product to $\mathbb{R}^{N}$ endowed with the scalar product $g$, and the map $Q^{+}\left(\mathbb{R}^{N}\right) \rightarrow \mathrm{GL}\left(\mathbb{R}^{N}\right), g \mapsto L^{g}$ is $\mathrm{C}^{\infty}$ (even real analytic).

Proof. We recall the well-known argument. We will construct $e_{i}^{g}, i=1, \ldots, N$ by induction on $i$, with the additional property that, for each $i=1, \ldots, N$, the subset $\left\{e_{1}^{g}, \ldots, e_{i}^{g}\right\}$ generates the same vector space as $\left\{e_{1}, \ldots, e_{i}\right\}$, where $e_{1}, \ldots, e_{N}$ is the canonical basis of $\mathbb{R}^{N}$. It suffices to take $e_{1}^{g}=e_{1} / \sqrt{g\left(e_{1}, e_{1}\right)}$. If $e_{1}^{g}, \ldots, e_{i-1}^{g}$ have already been defined, we denote by $f_{i}^{g}$ the orthogonal (with respect of the scalar product $g$ ) projection of $e_{i}$, on the subspace generated by $\left(e_{1}, \ldots, e_{i-1}\right)$. Since $\left(e_{1}^{g}, \ldots, e_{i-1}^{g}\right)$ is an orthonormal basis, for $g$, of this last vector subspace, we have

$$
f_{i}^{g}=\sum_{j=1}^{i-1} g\left(e_{i}, e_{j}^{g}\right) e_{j}^{g} .
$$

Obviously, the projection $f_{i}^{g}$ is, by induction, $\mathrm{C}^{\infty}$ (even real analytic) as a function of $g$. Since $e_{i}$ is not in the space generated by $\left\{e_{1}, \ldots, e_{i-1}\right\}$ (or $\left\{e_{1}^{g}, \ldots, e_{i-1}^{g}\right\}$ ), the vector $v_{i}^{g}=e_{i}-f_{i}^{g}$ is never 0 , therefore it suffices to set $e_{i}^{g}=v_{i}^{g} \sqrt{g\left(v_{i}^{g}, v_{i}^{g}\right)}$.

Proof of Proposition 2.26. Since continuity (resp. being locally Lipschitz) is a local property, we can assume that $M=U$ is an open subset of $\mathbb{R}^{N}$. Therefore, the tangent space $T M=T U$ is $U \times \mathbb{R}^{N}$. If we call $g_{x}$, the Riemannian metric on $T_{x} U=\{x\} \times \mathbb{R}^{N} \simeq \mathbb{R}^{N}$, applying Lemma 2.27 to $g_{x}$, we can define a map $L_{x}=$ $L^{g_{x}} \in \mathrm{GL}\left(\mathbb{R}^{N}\right)$ which is an isometry of $\mathbb{R}^{N}$ with the usual scalar product on $\mathbb{R}^{N}$ endowed with the scalar product $g_{x}$ and such that the map $U \rightarrow \operatorname{GL}\left(\mathbb{R}^{N}\right), x \mapsto L_{x}$ is $\mathrm{C}^{\infty}$. We have

$$
|v|=\left|L_{x}(v)\right|_{x} \quad \text { for any }(x, v) \in T M,
$$

which shows the first part of the proposition

Now we define $\tilde{\mathcal{C}}_{x}=L_{x}^{-1}\left(\mathcal{C}_{x}\right)$. By Lemma 2.19 , the family of cone $\tilde{\mathcal{C}}=\left(\tilde{\mathcal{C}}_{x}\right)$, $x \in U$, depends continuously on $x \in U$. By construction, we have for any $(x, v) \in T M$

$$
\begin{gathered}
d\left(v, \tilde{\mathcal{C}}_{x}\right)=d_{x}^{g}\left(L_{x} v, \mathcal{C}_{x}\right)=d_{\mathcal{C}}^{g}\left(x, L_{x} v\right), \\
d^{\#}\left(v, \tilde{\mathcal{C}}_{x}\right)=d_{x}^{g \#}\left(L_{x} v, \mathcal{C}_{x}\right)=d_{\mathcal{C}}^{g \#}\left(x, L_{x} v\right),
\end{gathered}
$$


where $d\left(x, \tilde{\mathcal{C}}_{x}\right)$ and $d^{\#}\left(v, \tilde{\mathcal{C}}_{x}\right)$ are the distance and the signed distance from $v$ to $\tilde{\mathcal{C}}_{x}$ measured in the usual Euclidean distance. Since the map $(x, v) \mapsto\left(x, L_{x} v\right)$ is a $\mathrm{C}^{\infty}$ diffeomorphism of $U \times \mathbb{R}^{N}$, to get the items i) and ii), it suffices to prove, that the maps $(x, v) \mapsto d\left(v, \tilde{\mathcal{C}_{x}}\right)$ and $(x, v) \mapsto d^{\#}\left(v, \tilde{\mathcal{C}}_{x}\right)$ are continuous (resp. locally Lipschitz). But this follows from Proposition 2.13. We pass to the proof of iii). Since $L_{x} \mathbb{B}=\mathbb{B}_{x}$, we obtain

$$
\begin{aligned}
I\left(\tilde{\mathcal{C}}_{x}\right) & =-\min _{v \in{\mathbb{B} \cap \tilde{\mathcal{C}}_{x}} d^{\#}\left(v, \tilde{\mathcal{C}}_{x}\right)} \\
& =-\min _{v \in \mathbb{B} \cap \tilde{\mathcal{C}}_{x}} d^{g \#}\left(L_{x} v, \mathcal{C}_{x}\right) \\
& =-\min _{w \in \mathbb{B}_{x} \cap \mathcal{C}_{x}} d_{\mathcal{C}}^{g \#}(x, w) \\
=I_{g}(\mathcal{C})(x) . &
\end{aligned}
$$

The continuity of $I_{g}(\mathcal{C})$ is now a consequence of Lemma 2.16.

To prove iv), we first argue that $L_{x}\left[\left(\tilde{\mathcal{C}}_{x}\right)^{-\delta}\right]=\mathcal{C}_{x}^{-\delta}$. In fact, for $v \in \mathbb{R}^{N}$, we have $L_{x}(v) \in \mathcal{C}_{x}^{-\delta}$ if and only if

$$
d_{\mathcal{C}}^{g \#}\left(x, L_{x} v\right) \leq-\delta\left|L_{x} v\right|_{x}
$$

By the computations made above this is the same as the inequality

$$
d^{\#}\left(v, \tilde{\mathcal{C}}_{x}\right) \leq-\delta|v|
$$

which precisely defines $\left(\tilde{\mathcal{C}}_{x}\right)^{-\delta}$.

Now if $\mathcal{C}^{\prime}$ is another cone structure on $M$, we obtain, arguing as above

$$
\max _{v \in \tilde{\mathcal{C}}_{x}^{\prime} \cap \mathbb{B}} d\left(v, \tilde{\mathcal{C}}_{x}\right)=\max _{w \in \mathcal{C}_{x}^{\prime} \cap \mathbb{B}_{x}} d_{x}^{g}\left(w, \mathcal{C}_{x}\right)=\max _{w \in \mathcal{C}_{x}^{\prime} \cap \mathbb{B}_{x}} d_{\mathcal{C}}^{g}(x, w)
$$

and, consequently

$$
\begin{aligned}
\delta^{H}\left(\tilde{\mathcal{C}}_{x}, \tilde{\mathcal{C}}_{x}^{\prime}\right) & =\max \left\{\max _{v \in \tilde{\mathcal{C}}_{x}^{\prime} \cap \mathbb{B}} d\left(v, \tilde{\mathcal{C}}_{x}\right), \max _{u \in \tilde{\mathcal{C}}_{x} \cap \mathbb{B}} d\left(u, \tilde{\mathcal{C}}_{x}^{\prime}\right)\right\} \\
& =\max \left\{\max _{w \in \mathcal{C}_{x}^{\prime} \cap \mathbb{B}_{x}} d_{\mathcal{C}}^{g}(x, w), \max _{z \in \tilde{\mathcal{C}}_{x} \cap \mathbb{B}_{x}} d_{\mathcal{C}^{\prime}}^{g}(x, z)\right\} \\
& =\delta_{x}^{g, H}\left(\mathcal{C}_{x}, \mathcal{C}_{x}^{\prime}\right) .
\end{aligned}
$$

In particular, this shows that

$$
\delta_{x}^{g, H}\left(\mathcal{C}_{x}^{-\delta}, \mathcal{C}_{x}\right)=\delta^{H}\left(\left(\tilde{\mathcal{C}}_{x}\right)^{-\delta}, \tilde{\mathcal{C}}_{x}\right)
$$

Since $\delta^{H}$ is the distance on Cones $\left(\mathbb{R}^{N}\right)$, we can now obtain iv) from the continuity of $(\delta, x) \mapsto\left(\tilde{\mathcal{C}}_{x}\right)^{-\delta}$ which follows from Lemma 2.17 .

Now the continuity of $(\delta, x) \mapsto\left(\tilde{\mathcal{C}}_{x}\right)^{-\delta}$ also implies that $\mathcal{C}^{-\theta}$ is a (continuous) cone structure for $\theta$ is continuous because

$$
\mathcal{C}_{x}^{-\theta(x)}=L_{x}\left(\left(\tilde{\mathcal{C}}_{x}\right)^{-\theta(x)}\right)
$$


Note that besides the continuity of $(\delta, x) \mapsto\left(\tilde{\mathcal{C}}_{x}\right)^{-\delta}$, Lemma 2.17 also shows that this map is locally Lipschitz if $\mathcal{C}$ is a Lipschitz cone structure and $\theta$ is continuous. Therefore $C^{-\theta}$ is a Lipschitz cone structure if $\theta$ is locally Lipschitz.

We now prove the last part of the Proposition. Since $\mathcal{C}_{x}^{\prime} \prec \mathcal{C}_{x}^{-\delta}$ if and only if $\tilde{\mathcal{C}_{x}^{\prime}} \prec\left(\tilde{\mathcal{C}_{x}}\right)^{-\delta}$, the fact that the $\left\{(\delta, x) \in\left[0,+\infty\left[\times M \mid \delta<I_{g}(\mathcal{C})(x), \mathcal{C}_{x}^{\prime} \prec \mathcal{C}_{x}^{-\delta}\right\}\right.\right.$ is open follows from Lemma 2.18.

We now proceed to extend theorem 2.15 to cone structures on manifolds.

Theorem 2.28. Let $\mathcal{C}$ be a (resp. Lipschitz) cone structure on the Riemannian manifold $M$. If $\epsilon: M \rightarrow] 0,+\infty[$ is a continuous function, we can find (resp. Lipschitz) cone structures $\mathcal{C}^{\prime} \prec \mathcal{C} \prec \mathcal{C}^{\prime \prime}$ with $\delta_{x}^{g, H}\left(\mathcal{C}_{x}, \mathcal{C}_{x}^{\prime}\right)<\varepsilon(x), \delta_{x}^{g, H}\left(\mathcal{C}_{x}, \mathcal{C}_{x}^{\prime \prime}\right)<$ $\varepsilon(x)$ for any $x \in M$.

Proof. As in Theorem 2.15, it is enough to show the existence of a cone structure $\mathcal{C}^{\prime}$ with the properties asserted in the statement, $\mathcal{C}^{\prime \prime}$ is in fact obtained from this by duality (more on this below).

The subset

$$
\mathcal{O}=\left\{( \delta , x ) \in \left[0,+\infty\left[\times M \mid \delta<I_{g}(\mathcal{C})(x), \delta_{x}^{g, H}\left(\mathcal{C}_{x}^{-\delta}, \mathcal{C}_{x}\right)<\varepsilon(x)\right\}\right.\right.
$$

is open in $[0,+\infty[\times M$, by item (iii) and (iv) of Proposition 2.26. It obviously contains $\{0\} \times M$, we can therefore find an open cover $\left(U_{i}\right)_{i \in I}$ of $M$ and a family $\left(\delta_{i}\right)_{i \in I}$ of (strictly) positive numbers such that $\{0\} \times M \subset \cup_{i \in I}\left[0, \delta_{i}\left[\times U_{i} \subset \mathcal{O}\right.\right.$. We take $\left(\theta_{i}\right)_{i \in I}$ a $\mathrm{C}^{\infty}$ partition of unity on $M$ subordinated to the open cover $\left(U_{i}\right)_{i \in I}$. The function $\theta$ defined by

$$
\theta(x)=\sum_{i \in I} \theta_{i}(x) \delta_{i}
$$

is $\mathrm{C}^{\infty}$, and $>0$. Moreover, we have $(\theta(x), x) \in \mathcal{O}$, for every $x \in M$. It follows from Proposition 2.26, that we can take $\mathcal{C}^{\prime}=\mathcal{C}^{-\theta}$.

To prove the existence of $\mathcal{C}^{\prime \prime}$ we argue by duality. We could just take a dual cone structure $\tilde{\mathcal{C}}^{\prime \prime} \prec \mathcal{C}^{*}$ with $\delta_{x}^{g, H}\left(\tilde{\mathcal{C}}_{x}^{\prime \prime}, \mathcal{C}_{x}^{*}\right)<\varepsilon(x)$, and take $\mathcal{C}^{\prime \prime}$ as the (pre-)dual of $\tilde{\mathcal{C}}^{\prime \prime}$. Note that instead of $\mathcal{C}^{*}$, we could use the (essentially same) cone structure $\mathcal{C}^{\sharp}$ on $M$ given by

$$
\mathcal{C}_{x}^{\sharp}=\left\{w \in T_{x} M \mid g_{x}(w, v) \leq 0, \forall v \in \mathcal{C}_{x}\right\} .
$$

This cone structure is simply $\mathcal{C}^{*}$ using the identification of $T M$ with $T^{*} M$ given by the Riemannian metric $g$

We get as a consequence:

Corollary 2.29. Given two continuous cone structures $\mathcal{C}, \mathcal{C}^{\prime \prime}$ on $M$ with $\mathcal{C}^{\prime \prime} \succ \mathcal{C}$, there exists a continuous cone structure $\mathcal{C}^{\prime}$ satisfying $\mathcal{C}^{\prime \prime} \succ \mathcal{C}^{\prime} \succ \mathcal{C}$. Moreover if either $\mathcal{C}$ or $\mathcal{C}^{\prime \prime}$ is a Lipschitz cone structure, we could choose $\mathcal{C}^{\prime}$ also Lipschitz. 
Proof. Keeping the notation of the proof previous proposition, we consider subset

$$
\mathcal{O}^{\prime}=\left\{( \delta , x ) \in \left[0,+\infty\left[\times M \mid \delta<I_{g}(\mathcal{C})(x), \mathcal{C}_{x}^{\prime \prime} \prec \mathcal{C}_{x}^{-\delta}\right\}\right.\right.
$$

By the last part of Proposition 2.26, this set is open in $[0,+\infty[\times M$. Moreover, it contains $\{0\} \times M$ since $\mathcal{C}^{\prime \prime} \prec \mathcal{C}$. Arguing as in the proof of the previous proposition, we can find a $\mathrm{C}^{\infty}$ function $\left.\theta: M \rightarrow\right] 0,+\infty[$ such that $(x, \theta(x)) \in$ $\mathcal{O} \cap \mathcal{O}^{\prime}$. We can then take $\mathcal{C}^{\prime}=\mathcal{C}^{-\theta}$.

Note that when $\mathcal{C}$ is Lipschitz, this gives a Lipschitz $\mathcal{C}^{\prime}$. If it is rather $\mathcal{C}^{\prime \prime}$ that it is Lipschitz, we could use duality to exchange the role of $\mathcal{C}$ and $\mathcal{C}^{\prime \prime}$.

\subsection{Adapted Cover and Metric}

It will simplify a lot the proofs if we can impose some technical conditions on Riemannian balls of a fixed radius.

We can always find a countable atlas of $\mathrm{C}^{\infty} \operatorname{charts} \varphi_{n}: U_{n} \rightarrow \mathbb{R}^{N}, n \in \mathbb{N}$ such that for each $n$

(i) The set $U_{n}$ is open and relatively compact in $M$;

(ii) the map $\varphi_{n}: U_{n} \rightarrow \mathbb{R}^{N}$ is a $\mathrm{C}^{\infty}$ diffeomorphism;

(iii) the family $\varphi_{n}^{-1}(\stackrel{\circ}{\mathbb{B}}), n \in \mathbb{N}$ covers $M$;

(iv) the family $\left(U_{n}\right)_{n \in \mathbb{N}}$ is a locally finite open covering of $M$.

It follows from the previous items that the set $\left\{m \in \mathbb{N} \mid U_{m} \cap U_{n} \neq \emptyset\right\}$ is finite for each $n \in \mathbb{N}$. We set $K_{n}=\varphi_{n}^{-1}(\mathbb{B})$ and $K_{n}^{\prime}=\varphi_{n}^{-1}(2 \mathbb{B})$. Therefore we get

$$
\begin{aligned}
& \bigcup_{n \in \mathbb{N}} K_{n}=M, \\
& K_{n} \subset \stackrel{\circ}{K_{n}^{\prime}} \subset K_{n}^{\prime} \subset U_{n}, \\
& \varphi_{n}\left(K_{n}^{\prime}\right) \text { is convex. }
\end{aligned}
$$

In the sequel of the paper, we will assume that the cover above has been chosen once and for all.

As we said before, we assume that $M$ is endowed with a Riemannian metric $g$. The next result tells us that $g$ can be modified, by multiplying it by a suitable smooth function, in order to obtain a complete Riemannian metric enjoying some additional properties.

Lemma 2.30. We can find a positive $\mathrm{C}^{\infty}$ function $\left.\beta: M \rightarrow\right] 0,+\infty[$ such that the Riemannian metric $\tilde{g}:=\beta^{2} g$ satisfies

$$
\bar{B}_{\tilde{g}}(x, 3)=\left\{y \in M \mid d^{\tilde{g}}(x, y) \leq 3\right\} \subset K_{n}^{\prime} \quad \text { for every } n \in \mathbb{N} \text {, every } x \in K_{n} .
$$


Proof. We set

$$
\delta_{n}=\min \left\{d_{g}(x, y) \mid x \in K_{n}, y \in \partial K_{n}^{\prime}\right\} .
$$

By compactness $\delta_{n}>0$. We claim that $\tilde{g}$ will satisfy the required property whenever $\beta: M \rightarrow] 0,+\infty[$ verifies

$$
\inf _{K_{n}^{\prime}} \beta \geq \frac{4}{\delta_{n}}
$$

In fact, the distance $\delta_{n}$ is nothing but the infimum of the lengths $\ell_{g}(\gamma)$ all curves $\gamma:[a, b] \rightarrow K_{n}^{\prime}$ with $\gamma(a) \in K_{n}, \gamma(b) \in \partial K_{n}^{\prime}$, and for such a curve $\gamma$ we have by (19)

$$
\ell_{\tilde{g}}(\gamma) \geq\left(\inf _{K_{n}^{\prime}} \beta\right) \ell_{g}(\gamma) \geq \frac{4}{\delta_{n}} \delta_{n}=4
$$

It follows that any point $y$ in $M$ at a distance strictly less than 4 for $\tilde{g}$ from a point in $K_{n}$ is necessarily in $K_{n}^{\prime}$.

It remains to construct $\beta$ such that $\inf _{K_{n}^{\prime}} \beta \geq 4 \delta_{n}^{-1}$, for all $n \in \mathbb{N}$. Notice that $\left\{m \in \mathbb{N} \mid U_{m} \cap U_{n} \neq \emptyset\right\}$ is finite, therefore we can set

$$
\lambda_{n}=\max \left\{\frac{4}{\delta_{m}} \mid m \in \mathbb{N}, U_{m} \cap U_{n} \neq \emptyset\right\}<+\infty .
$$

We fix a $C^{\infty}$ partition of unity $\left(\theta_{n}\right)_{n \in \mathbb{N}}$ subordinated to the open cover $\left(U_{n}\right)_{n \in \mathbb{N}}$, and we define

$$
\beta(x)=\sum_{n \in \mathbb{N}} \lambda_{n} \theta_{n}(x) .
$$

We have $\inf _{U_{n}} \beta \geq 4 \delta_{n}^{-1}$. In fact, if $x \in U_{n}$, and $\theta_{m}(x)>0$, we get $x \in U_{m} \cap U_{n}$. This implies $\lambda_{m} \geq 4 \delta_{n}^{-1}$, and therefore $\beta(x) \geq 4 \delta_{n}^{-1}$.

From now on we will assume that we have replaced $g$ by the Riemannian metric $\tilde{g}$. Therefore changing the name of $\tilde{g}$ back to $g$ we will assume

$$
\bar{B}_{g}(x, 3) \subset K_{n}^{\prime} \text { for every } x \in K_{n} .
$$

Note that the Riemannian metric $g$ is necessarily complete since the closed balls $B_{g}(x, 3)$ are compact for any $x \in M$.

\section{Intrinsic semi-distances and Aubry sets: Proof of Theorem 1.1}

We will assume that $\mathcal{C}$ is a (continuous) cone structure on $M$, which is endowed with the Riemannian metric $g$. We will also suppose that we have chosen a 
continuous function $\alpha: M \rightarrow] 0,+\infty\left[\right.$. We define the intrinsic length $\sigma_{g, \alpha}^{\mathcal{C}}(\gamma)$ of the Lipschitz curve $\gamma:[a, b] \rightarrow M$ by

$$
\sigma_{g, \alpha}^{\mathcal{C}}(\gamma)=\int_{\gamma} \alpha d_{\mathcal{C}}^{g}=\int_{a}^{b} \alpha(\gamma(t)) d_{\gamma(t)}^{g}\left(\dot{\gamma}(t), \mathcal{C}_{\gamma(t)}\right) d t
$$

The above quantity is nonnegative and invariant under orientation-preserving reparametrization of $\gamma$. Using intrinsic length of curves, we can introduce the intrinsic semi-distance $S_{\alpha, g}^{\mathcal{C}}$ on $M$, namely we set for $x, y$ in $M$

$$
S_{\alpha, g}^{\mathcal{C}}(x, y)=\inf _{\gamma} \sigma_{g, \alpha}^{\mathcal{C}}(\gamma)
$$

where the infimum is taken on all Lipschitz curves $\gamma$ joining $x$ to $y$. It is also clear that if $\mathcal{C}^{\prime}$ is another cone structure on $M$ with $\mathcal{C} \prec \mathcal{C}^{\prime}$ then $S_{\alpha, g}^{\mathcal{C}^{\prime}} \leq S_{\alpha, g}^{\mathcal{C}}$. We now check that $S_{\alpha, g}^{\mathcal{C}}$ is indeed a (not necessarily symmetric) nonnegative semi-distance.

Proposition 3.1. The nonnegative function $S_{\alpha, g}^{\mathcal{C}}$ satisfies

$$
\begin{aligned}
& S_{\alpha, g}^{\mathcal{C}}(x, x)=0, \\
& S_{\alpha, g}^{\mathcal{C}}(x, z) \leq S_{\alpha, g}^{\mathcal{C}}(x, y)+S_{\alpha, g}^{\mathcal{C}}(y, z) .
\end{aligned}
$$

Moreover, the function $S_{\alpha, g}^{\mathcal{C}}$ is locally Lipschitz on $M \times M$.

Proof. The fact that $S_{\alpha, g}^{\mathcal{C}}(x, x)=0$ follows from the homogeneity in $v$ of $d_{\mathcal{C}}^{g}(x, v)$ which implies that $\sigma_{g, \alpha}^{\mathcal{C}}(\gamma)=0$ for a constant curve $\gamma$. The triangle inequality $S_{\alpha, g}^{\mathcal{C}}(x, z) \leq S_{\alpha, g}^{\mathcal{C}}(x, y)+S_{\alpha, g}^{\mathcal{C}}(y, z)$ is a routine argument of concatenation of curves.

Since by the triangle inequality

$$
S_{\alpha, g}^{\mathcal{C}}\left(x^{\prime}, y^{\prime}\right)-S_{\alpha, g}^{\mathcal{C}}(x, y) \leq S_{\alpha, g}^{\mathcal{C}}\left(x^{\prime}, x\right)+S_{\alpha, g}^{\mathcal{C}}\left(y, y^{\prime}\right),
$$

it suffices to prove, to get the Lipschitz regularity of $S_{\alpha, g}^{\mathcal{C}}$, that for each $x_{0} \in M$, there is a neighborhood $V$ of $x_{0}$ a finite constant $C>0$ such that

$$
S_{\alpha, g}^{\mathcal{C}}\left(x^{\prime}, x\right) \leq C d^{g}\left(x, x^{\prime}\right) \quad \text { for all } x, x^{\prime} \in V .
$$

Since this is a local problem we can assume $M$ to be an open set in the Euclidean space $\mathbb{R}^{N}$, and $x_{0}=0$. We pick $r>0$ such that $V \subset r \mathbb{B} \subset M \subset \mathbb{R}^{N}$. By compactness

$$
C^{\prime}=\sup \left\{\alpha(x) d_{\mathcal{C}}^{g}(x, v)|x \in r \mathbb{B},| v \mid=1\right\}<+\infty .
$$

By homogeneity

$$
\alpha(x) d_{\mathcal{C}}^{g}(x, v) \leq C^{\prime}|v| \quad \text { for any } x \in r \mathbb{B}, v \in \mathbb{R}^{N} .
$$

Obviously, if $x, x^{\prime} \in r \mathbb{B}$ we consider the Euclidean segment $\xi(t)=(1-t) x^{\prime}+t x$, which satisfies $\xi(t) \in r \mathbb{B}$, and $\dot{\xi}(t)=x-x^{\prime}$, for any $t \in[0,1]$, to get

$$
S_{\alpha, g}^{\mathcal{C}}\left(x^{\prime}, x\right) \leq \sigma_{\alpha, g}^{\mathcal{C}}(\xi) \leq K^{\prime}\left|x-x^{\prime}\right|
$$

This finishes the proof since $d^{g}$ and the Euclidean distance are Lipschitz equivalent on $r \mathbb{B}$. 
We recall that any locally Lipschitz function on $M$ is differentiable for almost every (a.e. for short) $x \in M$, i.e. up to a subset of vanishing Riemannian measure. The intrinsic semi-distance is related to the dual cone structure $\mathcal{C}^{*}$ by the following proposition.

Proposition 3.2. Given $y \in M$, the locally Lipschitz functions $u_{y}=S_{\alpha, g}^{\mathcal{C}}(y, \cdot)$, and $v_{y}=S_{\alpha, g}^{\mathcal{C}}(\cdot, y)$ satisfy $D u_{y}(x) \in \mathcal{C}_{x}^{*},-D v_{y}(x) \in \mathcal{C}_{x}^{*},\left|D u_{y}(x)\right|_{x} \leq \alpha(x)$, and $\left|D v_{y}(x)\right|_{x} \leq \alpha(x)$ for a.e. $x \in M$.

Proof. The proof is taken from [7] with minor adjustments. We will do the proof for $u_{y}$. Consider a differentiability point $x \in M$ of $u_{y}$, it suffices to prove that

$$
D u_{y}(x) \in \mathcal{C}_{x}^{*} \quad \text { and } \quad\left|D u_{y}(x)\right|_{x} \leq \alpha(x) .
$$

Again this is a local result, therefore we can assume that $M$ is an open subset of $\mathbb{R}^{N}$. For $v \in \mathbb{R}^{n}$, and $\varepsilon>0$ small enough, the path $\gamma(t)=x+t \varepsilon v, t \in[0,1]$, is included in $M$, therefore

$$
\begin{aligned}
u_{y}(x+\varepsilon v)-u_{y}(x)= & S_{\alpha, g}^{\mathcal{C}}(y, x+\varepsilon v)-S_{\alpha, g}^{\mathcal{C}}(y, x) \\
& \leq S_{\alpha, g}^{\mathcal{C}}(x, x+\varepsilon v) \\
& \leq \sigma_{\alpha, g}^{\mathcal{C}}(\gamma)=\int_{0}^{1} \alpha(x+t \varepsilon v) d_{\mathcal{C}}^{g}(x+t \varepsilon v, \varepsilon v) d t \\
& =\varepsilon \int_{0}^{1} \alpha(x+t \varepsilon v) d_{\mathcal{C}}^{g}(x+t \varepsilon v, v) d t
\end{aligned}
$$

Therefore dividing by $\varepsilon>0$ and letting $\varepsilon \rightarrow 0$, we get

$$
D u_{y}(x)[v] \leq \alpha(x) d_{\mathcal{C}}^{g}(x, v)
$$

Since $d_{\mathcal{C}}^{g}(x, v)=d_{x}^{g}\left(v, \mathcal{C}_{x}\right) \leq|v|_{x}$, we obtain $\left|D u_{y}(x)\right|_{x} \leq \alpha(x)$. Moreover $d_{\mathcal{C}}^{g}(x, v) \leq$ 0 for $v \in \mathcal{C}_{x}$. Hence $D u_{y}(x)[v] \leq 0$ for $v \in \mathcal{C}_{x}$, which means $D u_{y}(x) \in \mathcal{C}_{x}^{*}$

The proof of Theorem 1.1 is based on the notion of Aubry set that we now introduce. Given a positive smooth function $\alpha$ on $M$, we define $\mathcal{A}_{\alpha, g}^{\mathcal{C}}$ as the set of points $x$ of $M$ such that there is a sequence of closed curves $\gamma_{n}$ based at $x$ with

$$
\inf _{n} \ell_{g}\left(\gamma_{n}\right)>0 \quad \text { and } \quad \inf _{n} \sigma_{\alpha, g}^{\mathcal{C}}\left(\gamma_{n}\right)=0
$$

The main point will be to show that $\alpha$ can be chosen in such a way that the corresponding Aubry set is empty.

The connection of this issue with the global time function we are looking for, is given by the following theorem which is a direct consequence of what is proved in [7], see in particular Theorem 6.2 there, and go back to the Introduction for more explanation. 
Theorem 3.3. If $\mathcal{A}_{\alpha, g}^{\mathcal{C}}$ is empty then there exists a $\mathrm{C}^{\infty}$ function $f: M \rightarrow \mathbb{R}$ such that $-D f(x) \in \mathcal{C}^{*}{ }_{x}$ and $|D f(x)|_{x} \leq \alpha(x)$ for every $x \in M$.

In other terms, $f$ is the sought global time function.

We refer to the Introduction for the definitions of causal and stably causal cone structure. Let $\mathcal{C}$ be a stably causal cone structure on $M$, we fix $\mathcal{C}^{\prime}$ a causal cone structure with $\mathcal{C}^{\prime} \succ \mathcal{C}$.

In the sequel we will assume that $\left(U_{n}, \varphi_{n}\right), K_{n}, K_{n}^{\prime}$ are the ones obtained at the beginning of section 2.5. We will further assume that the Riemannian metric $g$ on $M$ satisfies

$$
\bar{B}_{g}(x, 3) \subset K_{n}^{\prime} \text { for all } n \geq 0, x \in K_{n},
$$

as this was shown to be possible in Lemma 2.30 .

The next result, which essentially exploits Proposition 2.24, ensures that, for a suitable choice of $\alpha$, if the intrinsic length $\int_{\gamma} \alpha d_{g}^{\mathcal{C}}$ of a non-constant curve $\gamma:[a, b] \rightarrow M$ is small compared to its Riemannian length, then we can find a $\mathcal{C}^{\prime}$-future directed curve $\xi:[a, b] \rightarrow M$ with $\xi(a)=\gamma(a)$, and $\xi(b)=\gamma(b)$. In view of the causality assumption on $\mathcal{C}^{\prime}$, this implies that $\gamma$ cannot be a closed curve. Looking at the very definition of Aubry set above, we can, in turn, deduce that $\mathcal{A}_{\alpha, g}^{\mathcal{C}}=\emptyset$ and conclude the proof of Theorem 1.1 using Theorem 3.3. It is then left to show:

Theorem 3.4. We can find a $\mathrm{C}^{\infty}$ positive function $\left.\alpha: M \rightarrow\right] 0,+\infty[$ such that if $\gamma:[a, b] \rightarrow M$ is a curve with

$$
\sigma_{\alpha, g}^{\mathcal{C}}(\gamma)=\int_{\gamma} \alpha d_{g}^{\mathcal{C}}<\min \left\{\ell_{g}(\gamma), \frac{1}{2}\right\},
$$

then there exists a $\mathcal{C}^{\prime}$-future directed piecewise $C^{\infty}$ curve $\tilde{\gamma}:[a, b] \rightarrow M$ with $\tilde{\gamma}(a)=\gamma(a)$ and $\tilde{\gamma}(b)=\gamma(b)$. Moreover, at each $t \in[a, b]$, both left and right derivatives $\dot{\tilde{\gamma}}_{-}(t), \dot{\tilde{\gamma}}_{+}(t)$ are contained in $\dot{\mathcal{C}}_{\tilde{\gamma}(t)}^{\prime}$.

Proof. Note that the curve $\gamma$ cannot be constant, since it would have length 0 , and this would contradict $\sigma_{\alpha, g}^{\mathcal{C}}(\gamma) \geq 0$. To avoid heavy notations, we identify $K_{n}^{\prime}$, for any $n$, to a convex compact subset of $\mathbb{R}^{N}$ through the diffeomorphism $\varphi_{n}$. Using the function $d_{\mathcal{C}}$ obtained from the Euclidean distance in (14), by Proposition 2.24 we can find suitable positive constants $\tilde{\rho}_{n}, \tilde{\delta}_{n}$ such that for any Lipschitz curve $\xi:[a, b] \rightarrow K_{n}^{\prime}$ which satisfies

$$
\int_{\xi} d_{\mathcal{C}}<\min \left\{\tilde{\rho}_{n}, \tilde{\delta}_{n} \ell(\xi)\right\}
$$

where $\ell(\xi)$ is the Euclidean length of $\xi$, there exists a piecewise linear $\mathcal{C}^{\prime}$-future directed curve $\tilde{\xi}$ contained in $K_{n}^{\prime}$, joining $\xi(a)$ to $\xi(b)$. Notice that such a piecewise linear curve satisfies automatically the last part of the statement of the theorem concerning the right and left derivative. 
By compactness of $K_{n}^{\prime}$ we can find a positive number $a_{n}$ such that

$$
\frac{1}{a_{n}}|v| \leq|v|_{x} \leq a_{n}|v| \quad \text { for all } x \in K_{n}^{\prime}, v \in \mathbb{R}^{N} .
$$

Since $d_{\mathcal{C}}(x, \cdot)$ and $d_{\mathcal{C}}^{g}(x, \cdot)$ are distance functions to the same $\mathcal{C}_{x}$, this implies that

$$
\frac{1}{a_{n}} d_{\mathcal{C}}(x, v) \leq d_{\mathcal{C}}^{g}(x, v) \leq a_{n} d_{\mathcal{C}}(x, v) \quad \text { for all } x \in K_{n}^{\prime}, v \in \mathbb{R}^{N}
$$

It also implies that for any curve $\xi$ with support contained in $K_{n}^{\prime}$, we have

$$
\frac{1}{a_{n}} \ell(\xi) \leq \ell_{g}(\xi) \leq a_{n} \ell(\xi)
$$

where $\ell_{g}(\xi)$ is the Riemannian length of $\xi$.

We claim that for any Lipschitz curve $\xi:[a, b] \rightarrow K_{n}^{\prime}$ which satisfies

$$
\int_{\xi} d_{\mathcal{C}}^{g}<\min \left\{\frac{1}{a_{n}} \tilde{\rho}_{n}, \frac{1}{a_{n}^{2}} \tilde{\delta}_{n} \ell_{g}(\xi)\right\},
$$

there exists a piecewise linear $\mathcal{C}^{\prime}$-future directed curve $\tilde{\xi}$, with support contained in $K_{n}^{\prime}$, joining $\xi(a)$ to $\xi(b)$. This is true simply because (26) implies (22), as we now show in the following computation which exploits (24), (25)

$$
\begin{aligned}
\int_{\xi} d_{\mathcal{C}} & \leq a_{n} \int_{\xi} d_{\mathcal{C}}^{g}<a_{n} \min \left\{\frac{1}{a_{n}} \tilde{\rho}_{n}, \frac{1}{a_{n}^{2}} \tilde{\delta}_{n} \ell_{g}(\xi)\right\} \\
& \leq a_{n} \min \left\{\frac{1}{a_{n}} \tilde{\rho}_{n}, \frac{1}{a_{n}} \tilde{\delta}_{n} \ell(\xi)\right\}=\min \left\{\tilde{\rho}_{n}, \tilde{\delta}_{n} \ell(\xi)\right\}
\end{aligned}
$$

We set $\rho_{n}=\frac{\tilde{\rho}_{n}}{a_{n}}, \delta_{n}=\frac{\tilde{\delta}_{n}}{a_{n}^{2}}$, and we choose a $\mathrm{C}^{\infty}$ function $\left.\alpha: M \rightarrow\right] 0,+\infty[$ with $\alpha>\max \left\{\frac{1}{\delta_{n}}, \frac{2}{\rho_{n}}\right\}$ on $K_{n}^{\prime}$, for each $n \in \mathbb{N}$. Such a function can be constructed arguing as in Lemma 2.30 .

The rest of the proof goes along the same lines as that of Proposition 2.24, so we just give the main points of it. We assume $\gamma(a) \in K_{n_{1}}$, if $\gamma([a, b])$ is entirely contained in $B_{g}(\gamma(a), 1) \subset K_{n_{1}}^{\prime}$ then

$$
\begin{aligned}
\frac{1}{\delta_{n_{1}}} \int_{\gamma} d_{g}^{\mathcal{C}}<\int_{\gamma} \alpha d_{g}^{\mathcal{C}} \leq \ell_{g}(\gamma), \\
\frac{2}{\rho_{n_{1}}} \int_{\gamma} d_{g}^{\mathcal{C}}<\int_{\gamma} \alpha d_{g}^{\mathcal{C}} \leq \frac{1}{2},
\end{aligned}
$$

hence

$$
\int_{\gamma} \alpha d_{g}^{\mathcal{C}} \leq \min \left\{\frac{1}{a_{n_{1}}} \tilde{\rho}_{n_{1}}, \frac{1}{a_{n_{1}}^{2}} \tilde{\delta}_{n_{1}}\right\} .
$$


and the curve $\tilde{\gamma}$ exists by what we saw above. If instead the curve goes out from $B_{g}(\gamma(a), 1)$ then its first exit time from this set, say $t_{1}$, satisfies $d_{g}\left(\gamma(a), \gamma\left(t_{1}\right)\right)=1$ and we have

$$
\max \left\{\frac{2}{\rho_{1}}, \frac{1}{\delta_{1}}\right\} \int_{\left.\gamma\right|_{\left[a, t_{1}\right]}} d_{\mathcal{C}}^{g} \leq \int_{\gamma} \alpha d_{\mathcal{C}}^{g} \leq \frac{1}{2} \leq d_{g}\left(\gamma(a), \gamma\left(t_{1}\right)\right) \leq \ell_{g}\left(\left.\gamma\right|_{\left[a, t_{1}\right]}\right),
$$

from which we deduce, from what was obtained before, that we can find a piecewise linear $\mathcal{C}^{\prime}$-future directed curve $\tilde{\gamma}:\left[a, t_{1}\right] \rightarrow K_{n_{1}}^{\prime}$, with $\tilde{\gamma}(a)=\gamma(a)$ and $\tilde{\gamma}\left(t_{1}\right)=\gamma\left(t_{1}\right)$.

Then, still following the argument of Proposition 2.24, we construct by induction a finite sequence $t_{0}=a, t_{1}, \ldots, t_{k}=b$, and piecewise smooth $\mathcal{C}^{\prime}$-future directed curves $\tilde{\gamma}:\left[t_{j}, t_{j+1}\right] \rightarrow K_{n_{j}}^{\prime}$, with $\tilde{\gamma}\left(t_{j}\right)=\gamma\left(t_{j}\right)$ and $\tilde{\gamma}\left(t_{j+1}\right)=\gamma\left(t_{j+1}\right)$. In the final step, like in the proof of Proposition 2.24, we argue on $\left.\gamma\right|_{\left[t_{k-2}, t_{k}\right]}$, since the Riemannian length of $\gamma \mid\left[t_{k-1}, t_{k}\right]$ cannot be estimated from below.

We deduce from Theorem 1.1 a corollary which will be used in the proof of Theorems 1.2 and 1.3.

Corollary 3.5. Let $\mathcal{C}$ be a stably causal cone structure. For any compact subset $K \subset M$ there are two positive constants $\rho_{K}, l_{K}$ such that if $\mathcal{C}^{\prime}$ is any cone structure on $M$ satisfying $\max _{x \in K} \delta_{x}^{g, H}\left(\mathcal{C}_{x}, \mathcal{C}_{x}^{\prime}\right) \leq \rho_{K}$ then any $\mathcal{C}^{\prime}$-causal curve contained in $K$ has Riemannian length bounded from above by $l_{K}$.

Proof. By Theorem 1.1 there is $\mathrm{C}^{\infty}$ global time function $f$ for $\mathcal{C}$. Therefore, we can find $\theta>0$ such that

$$
D f(x)[v] \geq \theta \quad \text { for any } x \in K \text {, and any } v \in \mathcal{C}_{x} \text { with }|v|_{x}=1 .
$$

We define $m=\max _{K} f-\min _{K} f<+\infty$, and $A=\sup _{x \in K}|D f(x)|_{x}<+\infty$. We choose $\delta_{K}>0$ such that $\eta=\theta-2 A \rho_{K}>0$. Assume that $\max _{x \in K} \delta_{x}^{g, H}\left(\mathcal{C}_{x}, \mathcal{C}_{x}^{\prime}\right) \leq$ $\rho_{K}$. For $x \in K$, and $v^{\prime} \in \mathcal{C}_{x}^{\prime} \cap \partial \mathbb{B}_{x}$, by Lemma 2.11, applied to the Euclidean space $\left(T_{x} M,\|\cdot\|_{x}\right.$, we can find $v \in \mathcal{C}_{x} \cap \partial \mathbb{B}_{x}$ such that

$$
\left\|v^{\prime}-v\right\|_{x} \leq 2 \delta_{x}^{g, H}\left(\mathcal{C}_{x}, \mathcal{C}_{x}^{\prime}\right) \leq 2 \rho_{K} .
$$

It follows that

$$
\begin{aligned}
D f(x)\left(v^{\prime}\right) & \geq D f(x)(v)-\mid D f(x)\left\|_{x}\right\| v^{\prime}-v \|_{x} \\
& \geq \theta-2 A \rho_{K} \\
& =\eta>0 .
\end{aligned}
$$

In particular, if $\gamma:[0, \ell] \rightarrow K$ is a $\mathcal{C}^{\prime}$-causal curve parametrized by arc-length, we have

$$
D f(\gamma(t))(\dot{\gamma}(t)) \geq \eta \text {, for almost every } t \in[0, \ell] .
$$

Integrating on $[0, \ell]$ yields $f(\gamma(\ell))-f(\gamma(0)) \geq \eta \ell$. Since $\gamma(\ell), \gamma(0) \in K$. This implies $\eta \ell \leq m=\max _{K} f-\min _{K} f$. Therefore if we choose $l_{K}=m / \eta$, we indeed have $\ell_{g}(\gamma)=\ell \leq l_{K}$. 


\section{Proof of Theorem 1.2}

We recall from the introduction that a cone structure $\mathcal{C}$ defined in $M$ is called globally hyperbolic if it is stably causal and all $\mathcal{C}$-causal curves connecting two given points have support contained in a compact (possibly empty) subset of $M$.

We start with some lemmas.

The first one is a particular version of a quite well-known semi-continuity of action for convex functional. For the convenience of the reader we give a more elementary proof of the particular version we need using only Jensen's inequality.

Lemma 4.1. Let $K, \mathcal{C}, \mathcal{C}^{n}$ be a compact subset of $M$ and continuous cone structures, respectively, satisfying

$$
\max _{x \in K} \delta_{x}^{H}\left(\mathcal{C}_{x}, \mathcal{C}_{x}^{n}\right) \rightarrow 0, \text { as } n \rightarrow+\infty
$$

If $\gamma_{n}:[a, b] \rightarrow K$ is a sequence of equi-Lipschitz $\mathcal{C}^{n}$-causal curves uniformly converging in $[a, b]$ to some curve $\gamma$, then $\gamma$ is $\mathcal{C}$-causal.

Proof. Since the $\mathcal{C}$-causality is a local property, we can assume $K$ to be a compact subset of $\mathbb{R}^{N}$, and $\delta^{H}$ is the Hausdorff distance on cones obtained from the usual Euclidean norm. Since the sequence $\gamma_{n}$ is equi-Lipschitz, we can find a constant $L<+\infty$ such that

$$
\left|\dot{\gamma}_{n}(t)\right| \leq L
$$

for any $n$, and any $t \in[a, b]$ for which $\dot{\gamma}_{n}(t)$ exists.

The Lipschitz path $\gamma$ is differentiable almost everywhere. We will prove that at a point $\left.t_{0} \in\right] a, b\left[\right.$, at which the derivative $\dot{\gamma}\left(t_{0}\right)$ exists we have $\dot{\gamma}\left(t_{0}\right) \in \mathcal{C}_{\gamma\left(t_{0}\right)}$.

For this we fix $\epsilon>0$. By continuity of $\gamma$ and the cone structure $\mathcal{C}$ we can find $\delta>0$ such that

$$
\delta^{H}\left(\mathcal{C}_{\gamma(t)}, \mathcal{C}_{\gamma\left(t_{0}\right)}\right) \leq \epsilon, \text { for every } t \in\left[t_{0}-\delta, t_{0}+\delta\right] .
$$

Since $\max _{x \in K} \delta^{H}\left(\mathcal{C}_{x}, \mathcal{C}_{x}^{n}\right) \rightarrow 0$, as $n \rightarrow+\infty$, we can find $n_{\epsilon}$ such that

$$
\max _{x \in K} \delta^{H}\left(\mathcal{C}_{x}, \mathcal{C}_{x}^{n}\right) \leq \epsilon, \text { forall } n \geq n_{\epsilon} .
$$

By the continuity of $x \mapsto \mathcal{C}_{x}$ and the fact the $\gamma_{n}$ converges uniformly to $\gamma$, taking $n_{\epsilon}$ larger if necessary we can also assume that

$$
\delta^{H}\left(\mathcal{C}_{\gamma_{n}(t)}, \mathcal{C}_{\gamma(t)}\right) \leq \epsilon \text {, for every } n \geq n_{\epsilon} \text {, and every } t \in[a, b] .
$$

It follows that

$$
\delta^{H}\left(\mathcal{C}_{\gamma_{n}(t)}^{n}, \mathcal{C}_{\gamma\left(t_{0}\right)}\right) \leq 3 \epsilon, \text { for every } n \geq n_{\epsilon} \text {, and every } t \in\left[t_{0}-\delta, t_{0}+\delta\right] .
$$

Since by (28), we have $\left|\dot{\gamma}_{n}(t)\right| \leq L$, using Proposition 2.13 we get

$$
\left|d\left(\dot{\gamma}_{n}(s), \mathcal{C}_{\gamma_{n}(s)}^{n}\right)-d\left(\dot{\gamma}_{n}(s), \mathcal{C}_{\gamma\left(t_{0}\right)}\right)\right| \leq 3 L \epsilon
$$


for every $n \geq n_{\epsilon}$, and every $s \in\left[t_{0}-\delta, t_{0}+\delta\right]$ ant which $\dot{\gamma}_{n}(s)$ exists. Since $\dot{\gamma}_{n}(t) \in \mathcal{C}_{\gamma_{n}(t)}^{n}$, for almost every $t \in[a, b]$, we obtain

$$
d\left(\dot{\gamma}_{n}(s), \mathcal{C}_{\gamma\left(t_{0}\right)}\right) \leq 3 L \epsilon
$$

for every $n \geq n_{\epsilon}$, and almost every $s \in\left[t_{0}-\delta, t_{0}+\delta\right]$. Using that $v \mapsto d\left(v, \mathcal{C}_{\gamma\left(t_{0}\right)}\right)$ is convex, we obtain from Jensen's inequality that

$$
d\left(\frac{\int_{t}^{t^{\prime}} \dot{\gamma}_{n}(s) d s}{t^{\prime}-t}, \mathcal{C}_{\gamma\left(t_{0}\right)}\right) \leq \frac{\int_{t}^{t^{\prime}} d\left(\dot{\gamma}_{n}(s), \mathcal{C}_{\gamma\left(t_{0}\right)}\right) d s}{t^{\prime}-t} \leq 3 L \epsilon,
$$

for every $n \geq n_{\epsilon}$, and every $t, t^{\prime} \in\left[t_{0}-\delta, t_{0}+\delta\right]$, with $t<t^{\prime}$. Since $\gamma_{n}$ is Lipschitz, we know that $\int_{t}^{t^{\prime}} \dot{\gamma}_{n}(s) d s=\gamma_{n}\left(t^{\prime}\right)-\gamma_{n}(t)$. It follows that

$$
d\left(\frac{\gamma_{n}\left(t^{\prime}\right)-\gamma_{n}(t)}{t^{\prime}-t}, \mathcal{C}_{\gamma\left(t_{0}\right)}\right) \leq 3 L \epsilon
$$

for every $n \geq n_{\epsilon}$, and every $t, t^{\prime} \in\left[t_{0}-\delta, t_{0}+\delta\right]$, with $t<t^{\prime}$. We can now let $n \rightarrow+\infty$ to obtain

$$
d\left(\frac{\gamma\left(t^{\prime}\right)-\gamma(t)}{t^{\prime}-t}, \mathcal{C}_{\gamma\left(t_{0}\right)}\right) \leq 3 L \epsilon
$$

for every $t, t^{\prime} \in\left[t_{0}-\delta, t_{0}+\delta\right]$, with $t<t^{\prime}$. If we set $t=t_{0}$, and let $t^{\prime} \rightarrow t_{0}$, we obtain

$$
d\left(\dot{\gamma}\left(t_{0}\right), \mathcal{C}_{\gamma\left(t_{0}\right)}\right) \leq 3 L \epsilon
$$

Since $\epsilon>0$ was arbitrary, and $L$ is a constant that does not depend on $\epsilon$, we can let $\epsilon \rightarrow 0$ to obtain $d\left(\dot{\gamma}\left(t_{0}\right), \mathcal{C}_{\gamma\left(t_{0}\right)}\right)=0$, and therefore $\dot{\gamma}\left(t_{0}\right) \in \mathcal{C}_{\gamma\left(t_{0}\right)}$.

Lemma 4.2. Let $\mathcal{C}$ be a stably causal cone structure. For any compact subset $K \subset M$ and $\varepsilon>0$ there is a positive constant $\rho=\rho_{K, \varepsilon}$ such that if $\gamma:[a, b] \rightarrow K$ is a $\mathcal{C}^{\prime}$-causal curve and $\max _{x \in K} \delta_{x}^{H}\left(\mathcal{C}_{x}, \mathcal{C}_{x}^{\prime}\right) \leq \rho$ then there exists a $\mathcal{C}$-causal curve $\hat{\gamma}:[a, b] \rightarrow K$ with $\max _{[a, b]} d_{g}(\gamma(t), \hat{\gamma}(t)) \leq \varepsilon$.

Proof. The argument is by contradiction. We assume the existence of a $\varepsilon_{0}>0$, of a sequence $\mathcal{C}^{n}$ of cone structures with

$$
\max _{x \in K} \delta_{x}^{H}\left(\mathcal{C}_{x}, \mathcal{C}_{x}^{n}\right) \leq \frac{1}{n} \quad \text { for any } n
$$

and of a sequence $\gamma_{n}:\left[a_{n}, b_{n}\right] \rightarrow K$ of $\mathcal{C}^{n}$-causal curves such that for any $n$, any $\mathcal{C}$-causal curve $\gamma:\left[a_{n}, b_{n}\right] \rightarrow K$

$$
\max _{\left[a_{n}, b_{n}\right]} d_{g}\left(\gamma_{n}(t), \gamma(t)\right)>\varepsilon_{0}
$$

We know from Lemma 3.5 that for $n$ large enough, say $n>n_{0}$, the length of the $\mathcal{C}^{n}$-causal curves with support contained in $K$ are equibounded from above by 
some constant $l_{K}$. We therefore have, up to an orientation preserving change of parameters which does not affect the property of being a $\mathcal{C}^{n}$-causal curve, that all such curves, for $n>n_{0}$, are parametrized by a common interval $[a, b]=\left[a_{n}, b_{n}\right]$ and they are in addition equi-Lipschitz on it. Therefore, we can apply Ascoli's Theorem to the sequence $\gamma_{n}, n>n_{0}$, in order to obtain, up to a subsequence, a uniform limit $\hat{\gamma}$ in $[a, b]$. We derive from Lemma 4.1 that $\hat{\gamma}$ is a $\mathcal{C}$-causal curve, which is in contradiction with (29).

We now give a characterization of the global hyperbolicity.

Proposition 4.3. The global hyperbolicity property of $\mathcal{C}$ can be equivalently expressed by requiring that the union of the supports of all $\mathcal{C}$-causal curves with endpoints in a given compact subset of $M$ is compact.

This result is well known in Lorentzian Geometry, see for instance [10], and can be demonstrated along the same lines in our setting. We supply a proof for reader's convenience. Some definitions and two lemmata are preliminary. Given $x \in M$, we define

$$
\begin{aligned}
& J_{\mathcal{C}}^{+}(x)=\{y \in M \mid \text { there exists a } \mathcal{C} \text {-causal curve joining } x \text { to } y\} \\
& J_{\mathcal{C}}^{-}(x)=\{y \in M \mid \text { there exists a } \mathcal{C} \text {-causal curve joining } y \text { to } x\}
\end{aligned}
$$

Using these sets, the definition of global hyperbolicity of $\mathcal{C}$ can be expressed by requiring that $J_{\mathcal{C}}^{+}(x) \cap J_{\mathcal{C}}^{-}(y)$ has compact closure for all pair of points $x, y$ of $M$. Proposition 4.3 then asserts that this is equivalent to $J_{\mathcal{C}}^{+}(K) \cap J_{\mathcal{C}}^{-}(K)$ being compact for any compact subset $K$ of $M$, where $J_{\mathcal{C}}^{+}(K)=\cup_{x \in K} J^{+}(x)$ and $J_{\mathcal{C}}^{-}(K)$ is defined similarly.

Before proving Proposition 4.3, we establish two lemmas.

Lemma 4.4. Let $\mathcal{C}$ be a globally hyperbolic cone structure. For any $y, z$ in $M$ the set $J_{\mathcal{C}}^{+}(y) \cap J_{\mathcal{C}}^{-}(z)$ is compact, possibly empty.

Proof. Let $J_{\mathcal{C}}^{+}(y) \cap J_{\mathcal{C}}^{-}(z)$ be non-empty, and take a sequence $x_{n}$ belonging to it. There consequently exist $\mathcal{C}$-causal curves $\xi_{n}$ defined in $\left[a_{n}, b_{n}\right]$ with $\xi_{n}\left(a_{n}\right)=y$, $\xi_{n}\left(b_{n}\right)=z, \xi_{n}\left(t_{n}\right)=x_{n}$ for some $t_{n}$. By the hyperbolicity assumption all the $\xi_{n}$ have support contained in some compact set $K$, therefore, thanks to Corollary 3.5, they have equibounded Riemannian length.

We can therefore assume, up to a change of parameter which does not affect the property of being $\mathcal{C}$-causal, that such curves are equi-Lipschitz and are defined in a common interval $[a, b]$. By applying Ascoli Theorem we find that the $\xi_{n}$ uniformly converge, up to a subsequence, in $[a, b]$ to a curve $\xi$ which is still $\mathcal{C}$ causal by Lemma 4.1. Consequently the $x_{n}$ converge to some $x_{0}$ belonging to the support of $\xi$, since $\xi(a)=y$ and $\xi(b)=z$, we deduce that the limit point $x_{0}$ belongs to $J_{\mathcal{C}}^{+}(y) \cap J_{\mathcal{C}}^{-}(z)$. This ends the proof. 
Lemma 4.5. Given $x_{0} \in M$, there are sequences $z_{n}$ and $y_{n}$ converging to $x_{0}$ such that the sets $J_{\mathcal{C}}^{-}\left(z_{n}\right), J_{\mathcal{C}}^{+}\left(y_{n}\right)$ are both nonincreasing with respect to the inclusion

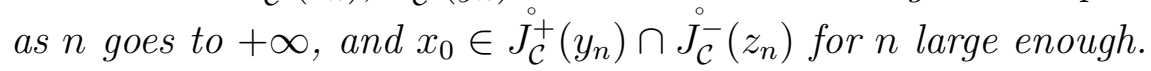

Proof. The argument is local in $x_{0}$, so we can assume to be in $\mathbb{R}^{N}$. By continuity of $\mathcal{C}$, we can determine a convex neighborhood $W$ of $x_{0}$ and an Euclidean unit vector $v_{0}$ with

$$
\sup _{x \in W} d^{\#}\left(v_{0}, \mathcal{C}_{x}\right)<0 .
$$

Therefore $t \mapsto x_{0}+t v_{0}$ is a $\mathcal{C}$-future directed curve for its part contained in $W$. We set $y_{n}=x_{0}-1 / n v_{0}$ and $z_{n}=x_{0}+1 / n v_{0}$ for $n$ so large that $y_{n}$ and $z_{n}$ are in $W$. By (30), up to further increasing $n$, the segments starting at(resp. ending to) points suitably close to $x_{0}$ and other endpoint at $z_{n}$ (resp. at $y_{n}$ ) are

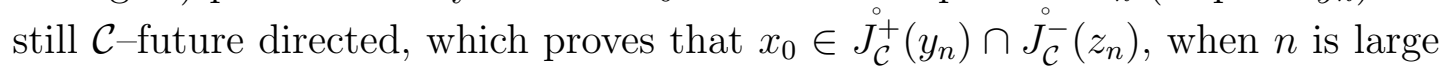
enough. Finally, the claimed monotonicity of $J_{\mathcal{C}}^{-}\left(z_{n}\right), J_{\mathcal{C}}^{+}\left(y_{n}\right)$ is apparent. This concludes the proof.

Proof of Proposition 4.3. One implication is clear. It is therefore enough to show that any globally hyperbolic cone structure possesses the property in the statement.

We first deduce from Lemmas 4.4 and 4.5 that $J_{\mathcal{C}}^{+}(x)$ and $J_{\mathcal{C}}^{-}(x)$ are closed for any $x$. Let a point $x_{0}$ be the limit of a sequence $x_{n}$ of $J_{\mathcal{C}}^{+}(x)$, since $x_{0} \in \stackrel{\circ}{\mathcal{C}}_{\mathcal{C}}^{-}(z)$ for a suitable $z$, then $x_{n} \in J_{\mathcal{C}}^{+}(x) \cap J_{\mathcal{C}}^{-}(z)$ when $n$ is large enough. Taking into account that $J_{\mathcal{C}}^{+}(x) \cap J_{\mathcal{C}}^{-}(z)$ is compact by Lemma 4.4, we infer that $x_{0} \in J_{\mathcal{C}}^{+}(x)$, as claimed. Same argument works for $J_{\mathcal{C}}^{-}(x)$.

The second step is to show that $J_{\mathcal{C}}^{+}(K)$ and $J_{\mathcal{C}}^{-}(K)$ are closed for any compact subset $K$ of $M$. We consider a point $x_{0}$ in the closure of $J_{\mathcal{C}}^{+}(K)$. Bearing in mind Lemma 4.5 and the fact that any neighborhood of $x_{0}$ intersects $J_{\mathcal{C}}^{+}(K)$, we can find a sequence $z_{n}$ converging to $x_{0}$ with $J_{\mathcal{C}}^{-}\left(z_{n}\right) \cap J_{\mathcal{C}}^{+}(K) \neq \emptyset$ and nonincreasing in $n$ with respect to the inclusion. This implies that $J_{\mathcal{C}}^{-}\left(z_{n}\right) \cap K$ are non-empty and, clearly, nonincreasing with respect to the inclusion. By the first part of the proof these sets are, in addition, compact and so possess a non-empty intersection.

If $z \in \cap_{n} J_{\mathcal{C}}^{-}\left(z_{n}\right) \cap K$ then $z_{n} \in J_{\mathcal{C}}^{+}(z)$ for any $n$ and, taking into account that $J_{\mathcal{C}}^{+}(z)$ is closed and $z \in K$, we find that $x_{0} \in J_{\mathcal{C}}^{+}(z) \subset J_{\mathcal{C}}^{+}(K)$. Similarly we prove the claim for $J_{\mathcal{C}}^{-}(K)$.

We can determine, by Lemma 4.5, for any $x \in K$ two points $y_{x}, z_{x}$ and an open neighborhood $V_{x}$ of $x$ with $V_{x} \subset J_{\mathcal{C}}^{-}\left(z_{x}\right) \cap J_{\mathcal{C}}^{+}\left(y_{x}\right)$. Since the $V_{x}$ make up an open covering of $K$, we can extract a finite subcover, and consequently determine two finite sets of points $\left\{y_{1}, \cdots, y_{h}\right\},\left\{z_{1}, \cdots, z_{h}\right\}$, for some $h$ in $\mathbb{N}$, such that $K \subset \cup_{i=1}^{h} J_{\mathcal{C}}^{+}\left(y_{i}\right), K \subset \cup_{j=1}^{h} J_{\mathcal{C}}^{-}\left(z_{j}\right)$, which implies $J_{\mathcal{C}}^{+}(K) \subset \cup_{i=1}^{h} J_{\mathcal{C}}^{+}\left(y_{i}\right)$, $J_{\mathcal{C}}^{-}(K) \subset \cup_{j=1}^{h} J_{\mathcal{C}}^{-}\left(z_{j}\right)$ and

$$
J_{\mathcal{C}}^{-}(K) \cap J_{\mathcal{C}}^{+}(K) \subset \cup_{i, j}\left(J_{\mathcal{C}}^{-}\left(z_{j}\right) \cap J_{\mathcal{C}}^{+}\left(y_{i}\right)\right) .
$$


Hence $J_{\mathcal{C}}^{-}(K) \cap J_{\mathcal{C}}^{+}(K)$ is contained in a compact subset of $M$ by Lemma 4.4 and so, being in addition closed by the previous step, it is in the end compact.

We come to the proof of Theorem 1.2.

We exploit Proposition 4.3 and define inductively an increasing sequence of compact domains $M_{n} \subset M$ with $\cup M_{n}=M$, by taking $M_{1}$ arbitrarily. We construct $M_{n}, n>1$ by induction. Suppose $M_{n-1}$ has been constructed, by Proposition 4.3, we can find a compact subset $K_{n}$, such that any $\mathcal{C}$-causal curve with endpoints in the compact subset $\bar{V}_{1}\left(M_{n-1}\right)=\left\{x \in M \mid d_{g}\left(x, M_{n-1}\right) \leq 1\right\}$ is entirely contained in $K_{n}$, we then set $M_{n}=\bar{V}_{1}\left(K_{n}\right)=\left\{x \in M \mid d_{g}\left(x, K_{n}\right) \leq 1\right\}$.

We denote by $N_{n}$ the compact subset

$$
\begin{array}{ll}
N_{n}=M_{n} & \text { for } n=1 \text { or } 2, \\
N_{n}=M_{n} \backslash \stackrel{\circ}{M}_{n-2} & \text { for } n>2 .
\end{array}
$$

It is clear that the $N_{n}$ are compact and constitute a locally finite covering of $M$. By Lemmas 3.5 and 4.2 we can determine, for any $n$, positive constants $l_{n}, \rho_{n}$, with $\rho_{n}$ decreasing, such that if a cone structure $\mathcal{C}^{\prime}$ satisfies $\sup _{x \in N_{n}} \delta_{x}^{H}\left(\mathcal{C}_{x}, \mathcal{C}_{x}^{\prime}\right) \leq \rho_{n}$ then:

(i) any $\mathcal{C}^{\prime}$-causal curve with support contained in $N_{n}$ has length estimated from above by $l_{n}$;

(ii) for any $\mathcal{C}^{\prime}$-causal curve $\gamma:[a, b] \rightarrow N_{n}$ there is a $\mathcal{C}$-causal curve $\hat{\gamma}:[a, b] \rightarrow$ $N_{n}$ with $\sup _{[a, b]} d_{g}(\gamma(t), \hat{\gamma}(t))<1$.

We can construct through Urysohn's Lemma a continuous function $\rho: M \rightarrow \mathbb{R}^{+}$ satisfying

$$
\max _{x \in N_{n}} \rho(x) \leq \rho_{n}
$$

We claim that any cone structure $\mathcal{C}^{\prime}$ satisfying

$$
\mathcal{C}^{\prime} \succ \mathcal{C} \quad \text { and } \quad \delta_{x}^{H}\left(\mathcal{C}_{x}, \mathcal{C}_{x}^{\prime}\right)<\rho(x) \text { for any } x
$$

is globally hyperbolic. We know from Theorem 2.28 that the family of cone structures satisfying both these properties is non-empty.

We proceed to prove that, for any $n$, any $\mathcal{C}^{\prime}$ satisfying (32), a $\mathcal{C}^{\prime}$-causal curve joining two points of $M_{n}$ is contained in $M_{n+1}$. In fact, if this is not the case, we find an $\bar{n}$ and one of such curves $\gamma$ joining two points of $\tilde{M}_{\bar{n}}$ with

$$
n_{0}:=\max \left\{n \mid \gamma \subset M_{n}\right\} \geq \bar{n}+2,
$$

so that there is a portion of $\gamma$, say $\gamma_{0}$, defined on the $[a, b]$, contained in $N_{n_{0}}=$ $M_{n_{0}} \backslash \stackrel{\circ}{M}_{n_{0}-2}$ with non-empty intersection with $M_{n_{0}} \backslash M_{n_{0}-1}$, and with its endpoints $\gamma_{0}(a), \gamma_{0}(b)$ in $\partial M_{n_{0}-2}$. 
Since

$$
\max _{x \in N_{n_{0}}} \rho(x) \leq \rho_{n_{0}}
$$

we find a $\mathcal{C}$-causal curve $\hat{\gamma}:[a, b] \rightarrow N_{n_{0}}$ with $\sup _{t \in[a, b]} d_{g}\left(\gamma_{0}(t), \hat{\gamma}(t)\right)<1$. Therefore the endpoints of $\hat{\gamma}$ are in $\bar{V}_{1}\left(M_{n_{0}-2}\right)=\left\{x \in M \mid d_{g}\left(x, M_{n_{0}-2}\right) \leq 1\right\}$, since $\gamma_{0}(a)$ and $\gamma_{0}(b)$ belong to $\partial M_{n_{0}-2}$. Therefore by the choice of $K_{n_{0}-1}$, the curve $\hat{\gamma}$ is contained in $K_{n_{0}-1}$, and $\gamma_{0}$ is contained in $\bar{V}_{1}\left(K_{n_{0}-1}\right)=\left\{x \in M \mid d_{g}\left(x, K_{n_{0}-1}\right) \leq\right.$ $1\}=M_{n_{0}-1}$. This is impossible since $\gamma_{0}$ has a point in $M_{n_{0}} \backslash M_{n_{0}-1}$.

We now claim that if $\mathcal{C}^{\prime}$ satisfies (32) then any $\mathcal{C}^{\prime}$-causal curve with support contained in a compact subset of $M$ has finite length. This implies that any such cone structures are causal and consequently stably causal since for any $\mathcal{C}^{\prime}$ it is possible to find $\mathcal{C}^{\prime \prime}$ still satisfying (32) and with $\mathcal{C}^{\prime \prime} \succ \mathcal{C}^{\prime}$.

To prove the claim it suffices to show that for any $n$, any $\mathcal{C}^{\prime}$-causal curve $\gamma$ contained in $M_{n}$ there exists a positive constant $l_{n}^{\prime}$ with $\ell_{g}(\gamma) \leq l_{n}^{\prime}$. The argument is by induction on $n$. The property is true for $M_{1}$ with $l_{1}^{\prime}=l_{1}$. Let $\gamma$, defined in $[a, b]$, be a $\mathcal{C}^{\prime}$-causal curve contained in $M_{n}$, if $\gamma$ is contained in $N_{n}$ then $\ell_{g}(\gamma) \leq l_{n}$ by (31). Otherwise there are points of $\gamma$ in $\stackrel{\leftrightarrow}{M}_{n-2}$, and we set

$$
\begin{aligned}
& a^{\prime}=\min \left\{s \in[a, b]: \gamma(s) \in M_{n-2}\right\} \\
& b^{\prime}=\max \left\{s \in[a, b]: \gamma(s) \in \tilde{M}_{n-2}\right\}
\end{aligned}
$$

Since $\left.\gamma\right|_{\left[a, a^{\prime}\right]}$ and $\left.\gamma\right|_{\left[b^{\prime}, b\right]}$, if not reduced to a point, are both contained in $N_{n}$, their length is estimated from above by $l_{n}$, thanks to (31). The $\mathcal{C}^{\prime}$-causal curve $\left.\gamma\right|_{\left[a^{\prime}, b^{\prime}\right]}$ connects two points of $M_{n-2}$ and so it is contained in $\tilde{M}_{n-1}$ by what we proved above, and, by inductive assumption, we know that $\ell_{g}\left(\left.\gamma\right|_{\left[a^{\prime}, b^{\prime}\right]}\right) \leq l_{n-1}^{\prime}$. We get in any case the estimate

$$
\ell_{g}(\gamma) \leq 2 l_{n}+l_{n-1}^{\prime}=: l_{n}^{\prime},
$$

which concludes the proof of the claim and of the theorem.

\section{Proof of Theorem 1.3}

Throughout the section we still denote by $g$ and $\alpha$ the Riemannian metric and the $\mathrm{C}^{\infty}$ positive function introduced in Section 3 , respectively. We indicate by $\mathcal{C}^{\prime}$ a globally hyperbolic cone structure with $\mathcal{C}^{\prime} \succ \mathcal{C}$. The existence of such an object is guaranteed by Theorem 1.2. Theorem 3.4 holds true and, in particular, $\mathcal{A}_{\alpha, g}^{\mathcal{C}}$ is empty. We will use a result following from [6, Theorem 8.5] or the proof of [7, Theorem 6.6]. For a cone structure, we can introduce, in the same way as we did for $\mathcal{C}$, the function $d_{\mathcal{C}^{*}}^{\# g}: \mathcal{T}^{*} M \rightarrow \mathbb{R}$ defined by

$$
\begin{aligned}
d_{\mathcal{C}^{*}}^{g \#}(x, p) & =2 d_{x}^{g}\left(p, \mathcal{C}_{x}^{*}\right)-d_{x}^{g}\left(v, \partial \mathcal{C}_{x}^{*}\right) . \\
& =\sup \left\{\left.p(v)\left|v \in \mathcal{C}_{x},\right| v\right|_{x}=1\right\} .
\end{aligned}
$$


This function is locally Lipschitz and convex in $p$. Moreover, for any $(x, p) \in$ $T^{*} M$, we have $p \in \mathcal{C}_{x}^{*}$ if and only if $d_{\mathcal{C}^{*}}^{g \#}(x, p)<0$.

Theorem 5.1. Consider a cone structure $\mathcal{C}$ on the manifold $M$. Assume $\mathcal{A}_{\alpha, g}^{\mathcal{C}}$ to be empty. Suppose that $\epsilon: M \rightarrow] 0,+\infty[$ is a continuous function If $h: M \rightarrow \mathbb{R}$ a locally Lipschitz function with $d_{\mathcal{C}^{*}}^{\# g}(x, D h(x)) \leq-\epsilon(x)$ for a.e. $x \in M$, we can construct a $\mathrm{C}^{\infty}$ function $\tilde{h}$ with $d_{\mathcal{C}^{*}}^{\# g}(x, D \tilde{h}(x) \leq-\epsilon(x) / 2$ for all $x \in M$ and, in addition, satisfying $\sup _{x \in M}|h(x)-\tilde{h}(x)| \leq 1$.

Proof. We introduce the Hamiltonian $H: T^{*} M \rightarrow \mathbb{R}$ defined by

$$
H(x, p)=\frac{d_{\mathcal{C}^{*}}^{\# g}(x, p)}{\epsilon(x)}
$$

It is not difficult to see that $H$ is continuous and convex in $p$. Moreover, we have $H(x, D h(x)) \leq-1$, for a.e. $x \in M$. Therefore by [6, Theorem 8.5] or the proof of $\left[7\right.$, Theorem 6.6], we can find a $\mathrm{C}^{\infty}$ function $\tilde{h}: M \rightarrow \mathbb{R}$ such that $H(x, D \tilde{h}(x)) \leq-1 / 2$, and $|h(x)-\tilde{h}(x)| \leq 1$, for every $x \in M$.

We will say that a $\mathcal{C}$-causal curve $\gamma$ defined in some interval $] a, b[$ is inextendible if both $\lim _{t \rightarrow a} \gamma(t), \lim _{t \rightarrow b} \gamma(t)$ do not exist. The first step is a characterization of inextendible $\mathcal{C}$-causal curves exploiting that $\mathcal{C}$ is stably causal.

Lemma 5.2. A $\mathcal{C}$-causal curve $\gamma$ defined on some interval $] a, b[$ is inextendible if and only if it does not admit any limit point for $t \rightarrow a$ and $t \rightarrow b$.

In particular, since we are assuming that the Riemannian metric on $M$ is complete, if the $\mathcal{C}$-causal curve $\gamma: I \rightarrow M$ is inextendible and parametrized by arc-length, then $I=]-\infty,+\infty[$.

Proof. Let $\gamma$ be $\mathcal{C}$-causal and inextendible. If the statement is false for $t \rightarrow b$, there are, by the very definition of inextendible curve, two distinct limit points $x$ and $y$, and one can select times $t_{1}<t_{2}<t_{3}$ in such a way that $\gamma\left(t_{1}\right), \gamma\left(t_{3}\right)$ are arbitrarily close to $x$ and $\gamma\left(t_{2}\right)$ is arbitrarily close $y$. We can form a closed loop $\xi$ based at $x$ by juxtaposition of the Riemannian geodesic between $x$ and $\gamma\left(t_{1}\right),\left.\gamma\right|_{\left[t_{1}, t_{3}\right]}$, and the Riemannian geodesic between $\gamma\left(t_{3}\right)$ and $x$. Without loss of generality, we can assume that the points $\gamma\left(t_{1}\right)$ and $\gamma\left(t_{3}\right)$ are contained in the compact Riemannian $\bar{B}(x, 1)$. It follows that we can find a constant $K$ such that

$$
\int_{\xi} \alpha d_{\mathcal{C}}^{g} \leq K\left[d_{g}\left(x, \gamma\left(t_{1}\right)\right)+d_{g}\left(x, \gamma\left(t_{3}\right)\right)\right]+\int_{\left.\gamma\right|_{\left[t_{1}, t_{3}\right]}} \alpha d_{\mathcal{C}}^{g}
$$

Since $\left.\gamma\right|_{\left[t_{1}, t_{3}\right]}$ is causal, the last term in the right hand side above is 0 , and

$$
\int_{\xi} \alpha d_{\mathcal{C}}^{g} \leq K\left[d_{g}\left(x, \gamma\left(t_{1}\right)\right)+d\left(x, \gamma\left(t_{3}\right)\right)\right]
$$


Therefore by choosing appropriately, we can assume $\int_{\xi} \alpha d_{\mathcal{C}}^{g}$ as small as we want. On the other hand $\ell_{g}(\xi) \geq 2 d_{g}\left(x, \gamma\left(t_{3}\right)\right)$. Since $\gamma\left(t_{3}\right)$ is as close as we want to $y$, we can assume that $\ell_{g}(\xi) \geq d_{g}(x, y)$. It follows that we can arrange $\xi$ to satisfy

$$
\int_{\xi} \alpha d_{\mathcal{C}}^{g}<\min \left\{\ell_{g}(\xi), \frac{1}{2}\right\} .
$$

This entails, in view of Theorem 3.4, that there exists a $\mathcal{C}^{\prime}$-future directed cycle based at $x$, which is impossible since $\mathcal{C}^{\prime}$ is causal.

The converse implication of the statement is immediate.

We break the body of the proof of Theorem 1.3 in two lemmas and a final part. We use Proposition 4.3 applied to $\mathcal{C}^{\prime}$ in the following construction of an increasing sequence of compact domains $W_{n}$.

The compact domains $W_{n} \subset M$, with $\cup_{i} W_{i}=M$, are defined taking $W_{1}$ arbitrarily but non-empty, and choosing, for $n>1, W_{n}=\bar{V}_{1}\left(K_{n}\right)=\{x \in M \mid$ $\left.d_{g}\left(x, K_{n}\right) \leq 1\right\}$, where $K_{n}$ is a compact subset containing $\bar{V}_{1}\left(W_{n-1}\right)=\{x \in M \mid$ $\left.d_{g}\left(x, W_{n-1}\right) \leq 1\right\}$, and such that any $\mathcal{C}^{\prime}$-causal curve connecting two points of $W_{n-1}$ is contained in $K_{n}$.

We will essentially use that $\mathcal{C}^{\prime} \succ \mathcal{C}$ is globally hyperbolic to show that the $W_{n}$ enjoy the crucial property given in the following:

Lemma 5.3. Let $y_{1}, y_{2}$ be in $W_{n-1}$, for some $n \geq 2$, and take $x \notin W_{n}$, then

$$
\max \left\{S_{\alpha, g}^{\mathcal{C}}\left(y_{1}, x\right), S_{\alpha, g}^{\mathcal{C}}\left(x, y_{2}\right)\right\} \geq 1 / 2
$$

Proof. We assume by contradiction

$$
S_{\alpha, g}^{\mathcal{C}}\left(y_{1}, x\right), S_{\alpha, g}^{\mathcal{C}}\left(x, y_{2}\right)<1 / 2,
$$

by the very definition of $W_{n}$ we have

$$
d_{g}\left(y_{1}, x\right), d_{g}\left(x, y_{2}\right)>1
$$

The inequalities (33), (34) imply, by Theorem 3.4, that there are $\mathcal{C}^{\prime}$-future directed curves from $y_{1}$ to $x$ and from $x$ to $y_{2}$, so that there exists a $\mathcal{C}^{\prime}$-causal curve connecting $y_{1}$ to $y_{2}$ and passing through $x$. From this we, in turn, derive that $x \in W_{n}$, in contradiction with the assumption.

We now consider a smooth function $\psi: M \rightarrow \mathbb{R}$ satisfying

$$
\begin{aligned}
& \psi \geq 2 \alpha \text { on } W_{1} \\
& \psi \geq 2 n \alpha \quad \text { on } W_{n} \backslash W_{n-1}, \text { for } n \geq 2 .
\end{aligned}
$$

We define $S_{\psi, g}^{\mathcal{C}}$ through formula (21) with $\psi$ in place of $\alpha$. Note that, since $\psi>\alpha$ on $M$, the Aubry set $\mathcal{A}_{\psi, g}^{\mathcal{C}}$ is empty, being $\mathcal{A}_{\alpha, g}^{\mathcal{C}}=\emptyset$ in force of Theorem 3.4. We deduce from the very definition of $\psi$ and Lemma 5.3 
Lemma 5.4. Let $\gamma:]-\infty,+\infty[$ be a $\mathcal{C}$-causal inextendible curve parametrized by the Riemannian arc-length, then

$$
\lim _{t \rightarrow+\infty} S_{\psi, g}^{\mathcal{C}}(\gamma(t), x)=\lim _{t \rightarrow-\infty} S_{\psi, g}^{\mathcal{C}}(x, \gamma(t))=+\infty
$$

for any fixed $x \in M$.

Proof. It suffices to show (35) for $x=\gamma(0)$, in fact by the triangle inequality

$$
S_{\psi, g}^{\mathcal{C}}(\gamma(t), x) \leq S_{\psi, g}^{\mathcal{C}}(\gamma(t), \gamma(0))+S_{\psi, g}^{\mathcal{C}}(\gamma(0), x)
$$

for any $t$, and a similar inequality applies to $S_{\psi, g}^{\mathcal{C}}(x, \gamma(t))$.

We select an $n$ so large that $\gamma(0) \in W_{n-1}$. By Lemma 5.2, $\gamma(t) \notin W_{n}$, whenever $|t|$ is sufficiently large. Given a $t_{0}>0$ enjoying such a property, we claim that

$$
S_{\psi, g}^{\mathcal{C}}\left(\gamma\left(t_{0}\right), \gamma(0)\right) \geq n
$$

To prove it, we fix $\varepsilon>0$, and select an $\varepsilon$-optimal curve $\xi:[a, b] \rightarrow M$ for $S_{\psi, g}^{\mathcal{C}}\left(\gamma\left(t_{0}\right), \gamma(0)\right)$. We set

$$
t_{1}=\min \left\{t \in[a, b] \mid \xi(t) \in W_{n-1}\right\} \quad \text { and } \quad \xi_{1}=\left.\xi\right|_{\left[a, t_{1}\right]} .
$$

We derive from Lemma 5.3 and the fact that $\gamma$ is $\mathcal{C}$-causal

$$
\frac{1}{2} \leq \max \left\{S_{\alpha, g}^{\mathcal{C}}\left(\gamma(0), \gamma\left(t_{0}\right)\right), S_{\alpha, g}^{\mathcal{C}}\left(\gamma\left(t_{0}\right), \xi\left(t_{1}\right)\right)\right\}=S_{\alpha, g}^{\mathcal{C}}\left(\gamma\left(t_{0}\right), \xi\left(t_{1}\right)\right) .
$$

Since $\xi_{1}$ lies outside $W_{n-1}$, we have $\int_{\xi_{1}} \psi \sigma_{g}^{\mathcal{C}} \geq 2 n \int_{\xi_{1}} \alpha \sigma_{g}^{\mathcal{C}}$, and we deduce from this inequality, the $\varepsilon$-optimality of $\xi$ and (37)

$$
\begin{aligned}
\frac{1}{2} & \leq S_{\alpha, g}^{\mathcal{C}}\left(\gamma\left(t_{0}\right), \xi\left(t_{1}\right)\right) \leq \int_{\xi_{1}} \alpha \sigma_{g}^{\mathcal{C}} \leq \frac{1}{2 n} \int_{\xi_{1}} \psi \sigma_{g}^{\mathcal{C}} \\
& \leq \frac{1}{2 n} \int_{\xi} \psi \sigma_{g}^{\mathcal{C}} \leq \frac{1}{2 n}\left(S_{\psi, g}^{\mathcal{C}}\left(\gamma\left(t_{0}\right), \gamma(0)\right)+\varepsilon\right)
\end{aligned}
$$

which gives (36), since $\varepsilon$ has been arbitrarily chosen. This, in turn, implies

$$
\lim _{t \rightarrow+\infty} S_{\psi, g}^{\mathcal{C}}(\gamma(t), x)=+\infty
$$

The same argument applies, with obvious adaptation, to prove the limit relation for $t$ going to $-\infty$.

We come to the final part of the proof of Theorem 1.3. To simplify notation, we fix some $x_{0}$, and we introduce the locally Lipschitz function $f_{1}: M \rightarrow \mathbb{R}$ defined by

$$
f_{1}(x)=S_{\psi, g}^{\mathcal{C}}\left(x, x_{0}\right)-S_{\psi, g}^{\mathcal{C}}\left(x_{0}, x\right) .
$$


By Proposition 3.2, we have $-D S_{\alpha, g}^{\mathcal{C}}\left(x, x_{0}\right) \in \mathcal{C}_{x}^{*}, D S_{\alpha, g}^{\mathcal{C}}\left(x_{0}, x\right) \in \mathcal{C}_{x}^{*}$ for a.e. $x$. It follows that $-D f_{1}(x) \in \mathcal{C}_{x}^{*}$ for a.e. $x \in M$. Moreover for any $\mathcal{C}$-causal curve $\gamma: I \rightarrow M$, the $t \mapsto f_{1}(\gamma(t))$ is non-decreasing, since this is the case for both $S_{\psi, g}^{\mathcal{C}}\left(\gamma(t), x_{0}\right)$ and $-S_{\psi, g}^{\mathcal{C}}\left(x_{0}, \gamma(t)\right)$. It therefore follows from Lemma 5.4, that for any inextendible $\mathcal{C}$-causal curve $\gamma:]-\infty,+\infty[\rightarrow M$, we have

$$
\lim _{t \rightarrow+\infty} f_{1}(\gamma(t))=+\infty \text { and } \lim _{t \rightarrow-\infty} f_{1}(\gamma(t))=-\infty .
$$

We now pick a $C^{\infty}$ global time function $f_{0}$ for $\mathcal{C}$ provided by Theorem 1.1 in correspondence to $\alpha$. We set

$$
f(x)=f_{0}(x)+f_{1}(x)
$$

Because $t \mapsto f_{0}(\gamma(t))$ is also non-decreasing along a $\mathcal{C}$-causal curve $\gamma$, we obtain that

$$
\lim _{t \rightarrow+\infty} f(\gamma(t))=+\infty \text { and } \lim _{t \rightarrow-\infty} f(\gamma(t))=-\infty,
$$

for any inextendible $\mathcal{C}$-causal curve $\gamma:]-\infty,+\infty\left[\rightarrow M\right.$. We have that $-D f_{0}(x) \in$ $\stackrel{\mathcal{C}}{x}_{x}^{*}$ for any $x \in$ and $-D f_{1}(x) \in \mathcal{C}_{x}^{*}$, or a.e. $x \in M$. Since $(x, p) \rightarrow d_{\mathcal{C}^{*}}^{g \#}(x, p)$ is convex and positively homogeneous in $p$, it is subadditive in $p$. We therefore get for a.e. $x \in M$

$$
\begin{aligned}
d_{\mathcal{C}^{*}}^{g \#}(x,-D f(x)) & \leq d_{\mathcal{C}^{*}}^{g \#}\left(x,-D f_{0}(x)\right)+d_{\mathcal{C}^{*}}^{g \#}\left(x,-D f_{1}(x)\right) \\
& \leq d_{\mathcal{C}^{*}}^{g \#}\left(x,-D f_{0}(x)\right) .
\end{aligned}
$$

Note that since $f_{0}$ is $\mathrm{C}^{1}$ and $-D f_{0}(x) \in \mathcal{C}_{x}^{*}$ for every $x \in M$, the function $\epsilon(x)=-d_{\mathcal{C}^{*}}^{g \#}\left(x,-D f_{0}(x)\right)$ is continuous and (strictly) positive everywhere on $M$.

Starting from $h=-f$, Theorem 5.1 finally allows us to find a smooth Cauchy time function going from $-\infty$ to $+\infty$ on any inextendible $\mathcal{C}$-causal curve, as claimed.

The proof of Theorem 1.3 is therefore concluded.

\section{References}

[1] J.P. Aubin, A. Cellina Differential Inclusion. Grundlehren der mathematischen Wissenschaften 264 Springer-Verlag, Berlin, 1984.

[2] J.K. Beem, P.E. Ehrlich \& K.L. Easley Global Lorentzian geometry. Monograph and Textbooks in Pure Appl. Math. 202 Marcel Dekker Inc., New York, 1996.

[3] G. BeER, Topologies on closed and closed convex sets, Kluwer Academic Publisher, Dordrecht, 1993 
[4] A.N Bernal, M. SÁnchez "On smooth Cauchy hypersurfaces and Geroch's splitting theorem". Comm. Math Phys, 243 (2003), 461-470.

[5] J. Dattorro, Convex optimization and Euclidean distance geometry, Meboo Publishing, USA, 2005.

[6] A. Fathi, E. Maderna "Weak KAM theorem on non compact manifolds", Nonlinear differ. equ. appl., 14 (2007), 1-27.

[7] A. Fathi, A. Siconolfi, "PDE aspects of Aubry-Mather theory for quasiconvex Hamiltonians", Calc. Var. Partial Differential Equations, 22 (2005), $185-228$.

[8] A. Fathi, Weak KAM Theorem in Lagrangian Dynamics, Cambridge University Press, to appear.

[9] R. Geroch, "Domain of dependence". J. Math. Phys 11 (1970), 437-449.

[10] S.W. Hawking, G.F.R ElLis The large scale structure of space-time. Cambridge Monographs on Mathematical Physics, Cambridge University Press, London-New York, 1973.

[11] B. O'NeILL Semi-Riemannian Geometry. Academic Press London-New York, 1983.

[12] A. Siconolfi, G. Terrone, "A Metric Approach to the Converse Lyapunov Theorem for Continuous Multivalued Dynamics", Nonlinearity, 20 (2007), 1077-1093.

Albert Fathi, Unité de mathématiques pures et appliquées, CNRS UMR 5669 \& École Normale Supérieure de Lyon, 46 allée d'Italie, 69364 Lyon, France afathi@umpa.ens-lyon.fr

Antonio Siconolfi, Dipartimento di Matematica, Università di Roma "La Sapienza". Piazzale A. Moro, 2, 00185 Roma, Italy

siconolf@mat . uniroma1. it 